\title{
DETERMINATION OF STABILITY AND CONTROL DERIVATIVES USING COMPUTATIONAL FLUID DYNAMICS AND AUTOMATIC DIFFERENTIATION
}

\author{
Michael A. Park ${ }^{*}$ \\ George Washington University \\ Joint Institute for the Advancement of Flight Sciences (JIAFS) \\ mikepark@tabdemo.larc.nasa.gov \\ Lawrence L. Green ${ }^{\dagger}$ \\ NASA Langley Research Center, Hampton, Virginia \\ 1.1.green@larc.nasa.gov \\ Raymond C. Montgomery \\ NASA Langley Research Center, Hampton, Virginia \\ r.c.montgomery@larc.nasa.gov \\ David L. Raney ${ }^{\S}$ \\ NASA Langley Research Center, Hampton, Virginia \\ d.1.raney@1arc.nasa.gov
}

\begin{abstract}
With the recent interest in novel control effectors there is a need to determine the stability and control derivatives of new aircraft configurations early in the design process. These derivatives are central to most control law design methods and would allow the determination of closed-loop control performance of the vehicle. Early determination of the static and dynamic behavior of an aircraft may permit significant improvement in configuration weight, cost, stealth, and performance through multidisciplinary design. The classical method of determining static stability and control derivatives - constructing and testing wind tunnel models - is expensive and requires a long lead time for the resultant data. Wind tunnel tests are also limited to the preselected control effectors of the model. To overcome these shortcomings, computational fluid

\footnotetext{
* Graduate Student, NASA Langley Research Center, Multidisciplinary Optimization Branch, MS 159, Hampton, Virginia, Member AIAA

${ }^{\dagger}$ Research Scientist, Multidisciplinary Optimization Branch, MS 159, Senior Member AIAA

${ }^{\ddagger}$ Research Scientist, Dynamics and Control Branch, MS 132, Senior Member AIAA

${ }^{\S}$ Research Scientist, Dynamics and Control Branch, MS 132, Member AIAA
}

Copyright $\odot 1999$ by the American Institute of Aeronautics, Inc. No copyright is asserted in the United States under Title 17, U.S. Code. The Government has royalty-free license to exercise all rights under the copyright claimed herein for government purposes. All other rights reserved by the copyright owner.
\end{abstract}

dynamics (CFD) solvers are augmented via automatic differentiation, to directly calculate the stability and control derivatives. The CFD forces and moments are differentiated with respect to angle of attack, angle of sideslip, and aircraft shape parameters to form these derivatives. A subset of static stability and control derivatives of a tailless aircraft concept have been computed by two differentiated inviscid CFD codes and verified for accuracy with central finite-difference approximations and favorable comparisons to a simulation database.

\section{Introduction}

Previous work attempted to determine stability derivatives from computational fluid dynamics (CFD) codes; for example, Finley ${ }^{1}$ used an Euler code to compute the forces and moments for a generic configuration from which a subset of the stability derivatives can be inferred by using finite-difference methods. Charlton ${ }^{2}$ employed a similar method on the Lockheed Martin Tactical Aircraft Systems-Innovative Control Effectors (LMTAS-ICE) ${ }^{\text {II }}$ configuration. No attempt was made to compute those derivatives analytically.

The present work proposes to use one or more CFD solvers, augmented via automatic differentiation (AD), to directly calculate static stability and control derivatives of the LMTAS-ICE configuration. Using exact AD is

\footnotetext{
${ }^{\mathrm{I}}$ The use of trademarks or names of manufacturers in this report is for accurate reporting and does not constitute an official endorsement, either expressed or implied, of such products or manufactures by the National Aeronautics and Space Administration
} 
more robust and removes the requirement of determining the optimal step size for the finite-difference calculation. This AD technique is illustrated with potential and Euler flow solvers, but can also be extended to Reynoldsaveraged Navier-Stokes (RANS) flow solvers to include viscous effects. The potential and Euler flow solver outputs (forces and moments) are differentiated with respect to the angle of attack and the angle of sideslip, yielding a subset of the static stability derivatives.

For control effectiveness, various control effectors can also be tested and optimized by finding the sensitivity of aircraft moments to changes in the configuration grid points that define these effectors. Because they are of greater importance to aircraft control, only moments were differentiated for control effectiveness, although the method is equally applicable to forces.

\section{Automatic Differentiation}

Automatic differentiation $^{3-5}$ is a technique for augmenting computer programs with statements for the computation of derivatives. It relies on the fact that every function, no matter how complicated, is executed on a computer as a (potentially very long) sequence of elementary operations such as additions, multiplications, and elementary functions such as sine and cosine. By repeatedly applying the chain rule of differential calculus to the composition of those elementary operations, derivative information can be computed exactly and in a completely automated fashion.

Two approaches for computing derivatives with $\mathrm{AD}$ are the forward mode and the reverse mode. The forward mode applies the chain rule of differentiation to propagate, equation by equation, derivatives of intermediate variables with respect to the input variables. In contrast, the reverse mode (adjoint) propagates, in reverse through the program, the derivatives of the output variables with respect to the input variables. The forward mode is more suited to problems with fewer input variables than output variables, whereas the reverse mode is better suited to problems with fewer output variables than input variables. Many hybrids of the forward and reverse modes are possible, with complementary tradeoffs in required random access memory (RAM), disk space, and execution time.

The forward method of $\mathrm{AD}$ is implemented in the ADIFOR $^{3}$ (Automatic Differentiation of FORTRAN) tool. The reverse mode is used in another tool, ADJIFOR $^{5}$ (Automatic Adjoint Generation in FORTRAN). The ADIFOR and ADJIFOR tools have been developed jointly by the Center for Research on Parallel Computation at Rice University and the Mathematics and Computer Sciences Division at Argonne National Laboratory. Both techniques are available in the prototype ADIFOR 3.0 package recently provided to the NASA Langley Research Center by Rice
University for alpha testing. In general, to apply ADIFOR or ADJIFOR to a given FORTRAN 77 code, the user is only required to specify those program variable names that correspond to the independent and dependent variables of the target differentiation. Each $\mathrm{AD}$ tool then determines the variables that require associated derivative computations, formulates the appropriate forward or reverse mode derivative expressions, and generates new FORTRAN 77 code for the computation of both the original simulation and the associated derivatives.

Currently, some manual processing is required to formulate the adjoint of iterative and parallel messagepassing codes due to the prototype nature of the alpha version of ADJIFOR used in this study. These manual manipulations of the code are expected to be greatly reduced with the formal release of the ADIFOR 3.0 package to the general public. This release is planned for the summer of 1999.

\section{Computational Fluid Dynamics Solvers}

The PMARC ${ }^{6}$ (Panel Method Ames Research Center) potential flow solver is a FORTRAN 77 code that can compute surface pressures, forces, and moments of arbitrary shapes. The code is based on the assumption of inviscid, irrotational, and incompressible flow, with some boundary layer and compressible corrections available, but not implemented in this study. PMARC also has a limited capability to compute solutions of unsteady, time-varying flow conditions.

The input file to the program includes the set of grid points describing the shape of the geometry as a set of panels. In this study, both the right and left halves of the LMTAS-ICE (Fig.1) configuration are modeled in the PMARC input file, with a total of 2560 panels. PMARC allows half the aircraft to be described and the solution to be mirrored in the $x-z$ plane. Although describing only one half of the configuration would reduce the time and memory required for a converged solution, this technique would not capture the effects of a nonzero angle of sideslip. The input file also specifies the flight condition, certain algorithmic parameters, and the user-defined position of the reference point about which all moments are summed. The forces and moments are also nondimensionalized with a user-specified reference area, length of the mean aerodynamic chord, and wingspan. All runs were performed with the assumption of incompressible flow or low-speed flight conditions.

The original PMARC code uses swap or scratch files to record intermediate values during operation. This disk usage allows PMARC to solve problems with a large number of panels on machines with limited RAM capacity. ADIFOR and ADJIFOR ignore FORTRAN read and write statements and are therefore unable to follow the dependency of variables through scratch file read and write operations. To allow the application of 
$\mathrm{AD}$, the PMARC code was modified to eliminate the need to use scratch files during execution. These modifications entailed replacing scratch file read and write operations with equivalent operations to common blocks during $\mathrm{AD}$ code generation. If sufficient RAM is not available for executing the code with the common blocks in the AD-generated code, the scratch file operations can be reactivated following code generation. To further minimize the use of RAM, scratch file operations for the new AD-created variables containing derivative information can also be inserted into the code in a fashion complementary to the original variables.

The forward mode of differentiation (ADIFOR) was used to compute the stability derivatives because there are two inputs - angle of attack and angle of sideslipcompared to the six output forces and moments. The reverse mode (ADJIFOR) is employed to calculate the derivatives used for guidance in the optimization of control effector size and placement, because the thousands of independent variables greatly outnumber the six dependent variables. The independent variables of these derivative calculations are the normal displacements of each of the surface-defining grid points. Normal displacements were chosen to mimic an inflatable device. The outputs are the three moment coefficients, each evaluated in three separate code executions.

Typical execution times for the original PMARC code are about four minutes on one R10000 processor of a Silicon Graphics Octane. The forward ADIFORgenerated PMARC aerodynamic analysis, including angle of attack and angle of sideslip derivatives, required approximately 12 minutes for the same single-processor execution scheme. The calculations were performed in 64-bit arithmetic with versions of codes that had all scratch file operations replaced with common blocks. The RAM requirements were 160 Mbytes for the PMARC function and 480 Mbytes for the function plus its derivatives with respect to angle of attack and angle of sideslip. The control derivatives of each separate moment with respect to the normal displacement of all the grid points is determined with the ADJIFOR reverse mode. This calculation was performed on the NASA Langley High Performance Computing and Communication Program (HPCCP) sixteen-processor Silicon Graphics Origin 2000 in 20 minutes with one processor. The memory requirements are 171 Kbytes RAM and 6 Gbytes of disk storage. Information from each of the over fifty iterations required to solve the aerodynamic interference coefficient matrix is stored to disk. This information is used to formulate the adjoint solution. The code can be modified to iterate the adjoint solution to save disk space with an increased cost in code execution time. ${ }^{5}$

The CFL3D (Computational Fluids Laboratory 3-Dimensional) code is a FORTRAN 77 Reynolds- averaged thin-layer Navier-Stokes flow solver for structured-volume grids. The analysis for this study was performed in an inviscid, Euler mode. The singleprocessor sequential version was differentiated into a version with angle of sideslip as the only independent in the derivative calculation. The code was only differentiated with respect to angle of sideslip to investigate a change in the simulation database derivatives between 5 and $7.5 \mathrm{deg}$ angle of attack. Work is ongoing to automatically differentiate the latest prerelease version of CFL3D. This work includes a parallel execution scheme that would allow faster derivative determination in both angle of attack and angle of sideslip. The volume grid used to model the airflow about both halves of the ICE configuration contains 3.1 million grid points.

The force and moment calculations were performed with a single processor of the HPCCP Silicon Graphics Origin 2000 with mesh sequencing and multigrid convergence acceleration on three levels. Timings are approximately 0.98 second per coarse-grid iteration, 18 seconds per medium-grid iteration, and 230 seconds per fine-grid iteration. The total time for a function evaluation was 18 hours. The sequential version required 825 Mbytes RAM. The inclusion of angle of sideslip derivatives caused a 500 percent increase in the sequential execution time and required 1600 Mbytes of RAM. The timing difference is possibly due to poor cache memory usage and AD overhead. This difference in execution time is further magnified by the use of aggressive compiler options, which are more effective on the original code. A parallel execution scheme employing 8 processors has demonstrated a 600 percent speedup from 18 to 3 hours for determining forces and moments. The differentiation of this parallel code may allow these stability derivatives to be calculated quickly enough to integrate this derivative determination into a design process.

\section{LMTAS-ICE}

The LMTAS-ICE is a proposed configuration that is used by NASA to test the synergy of low-observable technologies with new types of control effectors. ${ }^{7}$ The goal is a lightweight, low-observable, and highly maneuverable aircraft. The basic layout of the aircraft is a tailless, highly swept delta shape with integrated wing, body, and propulsion systems (see Fig.1). One of the goals of the LMTAS-ICE program is to investigate a wide range of conventional and novel control effectors for their impact on controllability, weight, and stealth.

The LMTAS-ICE configuration is a challenging aerodynamic problem for a potential flow solver such as PMARC because of a sharp leading edge and predicted high Mach numbers near the wing tips at cruise conditions. The combination of sharp leading edges and a highly swept planform can give rise to vortical flow at 
moderate to high angles of attack. Vortical flow is not modeled in the PMARC potential flow solver, but is modeled in the CFL3D Euler calculation. To further resolve these flow effects and to model some novel control effectors such as the synthetic jets, a fine-grid RANS solution may be required. PMARC analysis is most suited to the low angle of attack region (0 to $6 \mathrm{deg}$ ) of this aircraft's flight envelope, where the airflow is attached to the aircraft and where most cruise portions of a flight take place. This investigation was extended to an Euler code when the PMARC-calculated lateral derivatives showed nonideal (possibly unacceptable) correlation with the simulation database at larger angles of attack. Euler calculations may be more suited to these higher angles of attack and angles of sideslip (6 to 15 deg). Highly separated and time-varying flow conditions (>15 deg) may require the investment of time-dependent RANS calculations to correctly predict derivatives.

In spite of these concerns, PMARC was chosen for the initial work in this study because of its potential for rapid code execution and the option to solve either steady or unsteady flows. The LMTAS-ICE was chosen because of the existence of a wind-tunnel-based aerodynamic database and a desire to optimize control effectors for the configuration. There is also a desire to develop a "seamless" aircraft control scheme, ${ }^{7}$ where aircraft attitude is controlled with slight mold-line distortions rather than with hinged moving surfaces. These novel control effectors are designed to keep the flow attached and therefore lend themselves to the linear aerodynamic theory of PMARC. The ADJIFOR modification of CFD code is well suited to the task of finding guidance for the optimal definition of these continuous shape-morphing controls.

\section{Validation of AD by Central Finite Differences}

The first step in verifying the accuracy of the ADIFOR-generated version of PMARC was to compare the forces and moments of the ADIFOR-generated PMARC code to the forces and moments of the original version of PMARC. This comparison showed no significant discrepancies. The derivatives were then checked for accuracy using second order central-difference approximations. The cruise condition of $4.39 \mathrm{deg}$ angle of attack $(\alpha), 0 \mathrm{deg}$ angle of sideslip $(\beta)$, and 0.60 Mach was chosen as the point about which to perform the finite differencing. By, treating the forces and moments PMARC outputs as a function $(P)$ of angle of attack or sideslip $(A)$, the central-difference value of the force or moment derivative is calculated as

$$
\frac{\partial P}{\partial A}=\frac{P(A+\Delta)-P(A-\Delta)}{2 \Delta}+O\left(\Delta^{2}\right)
$$

The deltas $(\Delta)$, changes in angle of attack and sideslip, are chosen between 0.0001 and 5 deg (see Table $1)$. The value of the computed derivative is plotted as a function of the logarithm of $\Delta$ (Fig. 2). The dashed horizontal line is the ADIFOR-computed derivative. The symbols $C N, C A$, and $C S$ represent the nondimensional coefficients of aerodynamic forces referenced to the body axes in the directions upward, aft, and toward the right wing tip as shown in Fig.1. Figure 2a, 2b, and $2 \mathrm{~d}$ show a comparison of the force derivatives. The symbols $\mathrm{Cl}$, $\mathrm{Cm}$, and $\mathrm{Cn}$ represent the nondimensional coefficients of moments in the roll, pitch, and yaw axes as shown in Fig.1. Figure 2c, 2e, and $2 \mathrm{f}$ show the moment derivative comparisons.

For small $\Delta$, the central-difference calculations are equal to the ADIFOR values of the static stability derivatives for more than six significant figures. The difference between the central-difference approximation and ADIFOR derivative value grows larger with $\Delta$. A $\log$ scale for $\Delta$ was used in the figures. In this ADIFOR study, the central-differences are relatively insensitive to the size of $\Delta$. Previous ADIFOR finite-difference

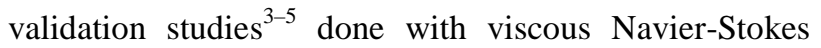
calculations showed a much greater sensitivity to the $\Delta$ size. This sensitivity is possibly due to both the nonlinear natures of the transonic Navier-Stokes codes for the problems investigated and the uses of first order onesided finite-difference approximations in the previous studies. One-sided finite-difference or central-difference approximation comparisons were not made to the ADIFOR-generated CFL3D Euler derivatives because of the computer time required for the many lengthy executions. Instead, the validity of the derivatives was inferred by direct comparisons to PMARC and wind-tunnel-based derivatives. Each PMARC central-difference approximation required four executions (two angle of attack and two angle of sideslip perturbations) of the PMARC code. Therefore, the time required for the code execution of a central-difference approximation was approximately 16 minutes. In contrast, all the derivatives can be computed in a single run (about 12 minutes) of the ADIFOR-generated PMARC code. This difference in processing time required for these two approaches will

become more significant in problems with more independent variables or more time-consuming, higher fidelity flow calculations.

\section{Force and Moment Comparison to Simulation Database}

The ADIFOR application of PMARC has been shown to produce the correct derivatives of its calculated forces and moments. The ADIFOR-generated PMARC and CFL3D codes are now further verified by comparing these results to the simulation database derived from 
wind tunnel data (see Fig. 3 and Tables 2-4). Note that the data at angle of sideslip not equal to zero (Tables 3 and 4) is shown as the difference between the displaced angle of sideslip $(\beta)$ data and data taken at zero angle of sideslip for the same angle of attack. All calculations were performed at a Mach number of 0.60 .

The wind tunnel data that is presented for comparison to the PMARC results was taken from an aerodynamic database used for a flight simulator. The way that the wind tunnel data was gathered and reduced to form the database needs to be researched. Further understanding of the methods used to convert the wind tunnel data into the simulation aerodynamic database may help to explain some of the differences between the aerodynamic database and PMARC in the lateral coefficients ( $C S, C l$, and $C n$ Fig. 3d-3f). These differences could also be highlighting flow properties not modeled in PMARC.

In an effort to improve the correlation between wind tunnel data and the values from PMARC and CFL3D, an estimate was sought of the drag component missing from the inviscid code calculations. This delta coefficient of drag $\left(\triangle C D_{0}\right)$ was estimated by subtracting the axial force coefficient $(C A)$ of the wind tunnel data from the corresponding value in PMARC when the normal force coefficients of both are near zero (see Fig. 3b). The $\Delta C D_{0}$ calculation is made when the normal force is near zero so that any lift-induced drag component of $\triangle C D_{0}$ is minimized. In the absence of wind tunnel data, an estimate of $\triangle C D_{0}$ can be based on theoretical skin friction calculations. The $\triangle C D_{0}$ oriented along the wind vector is resolved into the $C A, C N$, and $C S$ directions for all angles of attack and angles of sideslip. The force and moment reference center for the configuration is located a distance $(\Delta Z)$ below the centroid of lateral area, where the $\triangle C D_{0}$ is assumed to act. Therefore, the $\triangle C D_{0}$ increment to the forces also causes additional moments. The equations used to increment the various PMARC and CFL3D forces and moments shown in Fig. 4 and Tables 5a-5c are as follows: ${ }^{8}$

$$
\begin{aligned}
& \Delta C D_{0}=0.00868 \\
& \text { MAC }=28.75 \mathrm{ft} \quad \text { Span }=37.5 \mathrm{ft} \quad \Delta Z=9.0 \mathrm{ft} \\
& \alpha=\text { Angle of Attack } \quad \beta=\text { Angle of Sideslip } \\
& C N=C N+\sin \alpha \times \Delta C D_{0} \\
& C A=C A+\cos \alpha \times \Delta C D_{0} \\
& C S=C S-\cos \alpha \times \sin \beta \times \Delta C D_{0} \\
& C l=C l-\Delta Z / \operatorname{Span} \times \cos \alpha \times \sin \beta \times \Delta C D_{0} \\
& C m=C m+\Delta Z / \mathrm{MAC} \times \cos \alpha \times \Delta C D_{0} \\
& C n=C n
\end{aligned}
$$

Following the addition of these increments, the PMARC values show excellent trend agreement up to 6 deg angle of attack. At higher angles of attack the presumed predominance of vortical flow patterns starts to affect the lateral-directional coefficients dramatically. (Lateral displacements to nonzero angles of sideslip were not performed with CFL3D, but its accuracy can be inferred from the angle of sideslip derivatives computed with ADIFOR.) In contrast, CFL3D correctly predicted the change in lift slope, including changes due to leading edge vortical flow. Note that a $\Delta C D_{0}$ for CFL3D could also be estimated independent of the calculation done for PMARC, but this estimation was not performed due to the similarities between the results of these two codes.

\section{Derivative Comparison to Simulation Database}

The longitudinal forces and moments shown in Fig. 3 are differentiated with respect to angle of attack, and the lateral forces and moments are differentiated with respect to angle of sideslip. These derivative values are shown in Fig. 5. The derivatives plotted for PMARC are the ADgenerated values. The CFL3D derivatives are central-difference approximations for the longitudinal cases, and $\mathrm{AD}$-generated values for the lateral cases. (Work is under way for AD-generated CFL3D longitudinal derivatives, but these derivatives were not available in time for this publication.) Second order central differencing is performed on the simulation database between 2 deg positive and 2 deg negative angle of sideslip for the lateral cases, and a weighted central method is used for angle of attack derivatives because of the nonuniform spacing between points in the longitudinal cases. CFL3D provides a better prediction of the normal force slope (Fig. 5a), but both CFL3D and PMARC have difficulty with the pitching moment derivative (Fig. 5c), possibly indicating the need for a viscous Navier-Stokes calculation. ADIFOR-generated CFL3D lateral derivatives are similar to the ADIFORgenerated PMARC values at 2.5 and $5 \mathrm{deg}$ angle of attack. The lateral derivatives of the ADIFOR-generated CFD codes agree reasonably well with wind tunnel data below 6 deg angle of attack; then at higher angles of attack the wind tunnel data changes radically while PMARC remains linear.

The ADIFOR-generated CFL3D code converged to values near those of the wind tunnel at $7.5 \mathrm{deg}$ angle of attack for the coarse and medium grids. The $7.5 \mathrm{deg}$ angle of attack fine-grid derivatives converged to significantly different values (Fig 5e-5f), possibly indicating the simulation of different flow physics on this finer mesh than the two coarser meshes. The prediction that different flow phenomenon are being modeled is based on truncation error arguments. The truncation error of a CFD code is based on the difference between the discretized partial differential equation representing the flow field in the code and the original continuous partial 
differential equation. If the CFD solution is converging to the same exact solution as the mesh is refined, the truncation error of a CFD solution should decrease in a predictable manner. Because CFL3D is based on a second order method, the truncation error should vary linearly with a square of a characteristic length of the mesh. The forces, moments, and their derivatives are computed in the ADIFOR-generated CFL3D code as a function of the discretized flow equations. Therefore, The forces, moments, and their derivatives should vary in a predictable second order manner as the mesh is refined.

The CFL3D-calculated forces, moments, and derivatives at lower angles of attack converged in a predictable manner as the mesh size was sequenced. The forces and moments of the fine grid at $7.5 \mathrm{deg}$ angle of attack converged to values indicative of a second order reduction in truncation error, whereas the derivatives did not. Therefore, this flight condition of $7.5 \mathrm{deg}$ angle of attack is still under investigation.

The heightened sensitivity of lateral force and moment coefficients to angle of sideslip (particularly at angle of sideslip equal to zero) at this higher angle of attack can also be seen in the simulation database. (This data is not shown here due to paper length constraints.) The wind tunnel measurements, from which this simulation database was derived, were performed every 2 deg, between positive and negative $10 \mathrm{deg}$ angle of sideslip. Therefore, the angle of sideslip derivatives at angle of sideslip equal to zero were computed between positive and negative $2 \mathrm{deg}$ angle of sideslip or a finite-difference step of $4 \mathrm{deg}$. The forces and moments exhibit strongly nonlinear behavior as angle of sideslip is varied at 7.5 deg angle of attack.

Finite-difference approximations of the lateral derivatives in the simulation database can be interpreted to be very sensitive to step size and to the angle of sideslip about which the derivative approximation is centered, due to the nonlinear nature of the wind tunnel data. Using a finite-difference method with a large step size ( $4 \mathrm{deg}$ ) masks the actual behavior of these nonlinear functions of angle of sideslip. From previous experience, it is expected that the AD-generated derivatives for CFD codes that simulate the required flow physics present in the full scale or wind tunnel models will prove to be more accurate than derivatives of wind tunnel measurements obtained by coarse finite differences.

These Euler derivative calculations are expensive executions in terms of CPU time, but these processes may be more applicable to the design process if speed improvement can be achieved by increased use of RAM caching or parallel code execution. This derivative information may also be combined with function information to form response surfaces. Splines or other interpolation methods can be used to structure these response surfaces from the high-fidelity flow simulation data. These response surfaces can be used in the design process. The use of these interpolation methods, forced to satisfy the values of the function and its derivatives, may allow the calculations to be performed at fewer flight conditions and still have the same resolution of aircraft forces and moments, as more numerous, function-only calculations.

\section{PMARC Control Effectiveness Derivatives}

In this study, ADJIFOR-generated control effectiveness derivatives plotted as a function of control placement are used to provide guidance for the optimal placement of shape change control effectors. The shape of the aircraft is described in the PMARC input file as a set of panels, each designated by four corner grid points. The shape is modified by moving each of these grid points in and out a small amount along a vector normal to the surface. The derivative of the forces and moments of the configuration is calculated with respect to the normal displacement of these grid points. Optimal placement of these controls is located in areas of large gradients of the forces and moments. The placement of these effectors must allow for coordinated control of all three moments simultaneously because they may be highly coupled. A deployment scheme that allows the control force to be applied in a linear fashion with input command would be desirable from a pilot or control designers perspective, because the control force of these effectors can be very nonlinear with deflection height and simultaneous application of nearby effectors. Identifying the optimal locations would permit a certain deflection of the skin to have the largest effect on aircraft control. Control effectors that may be used in this manner are flexible, inflatable surfaces, shape memory alloys, and piezoelectrics.

This study focuses on the three moment coefficients because of their greater importance to closed-loop controller design. Figure 6 shows the ADJIFORgenerated values of the control effectiveness contours interpolated over the aircraft surface. Figure $6 a-6 c$ shows the control effectiveness of an upper surface deflection on the three moments in the roll, pitch, and yaw axes, respectively. Figure $6 \mathrm{e}-6 \mathrm{f}$ shows the control effectiveness of deflecting the lower surface of the configuration on the same three moments. Areas shaded in black and white offer the greatest amount of control effectiveness, but should be avoided because they often located near each other indicating an unacceptable level of sensitivity to effector location. Areas with colors in the red to pink range have larger contiguous areas, allowing a number of grid points to work together to generate a positive change in moment. Areas with colors ranging from blue to violet offer the same benefits for generating negative moments. The red and violet areas of the aircraft leading edge and slightly aft of the middle of the wing are currently being targeted for effector placement. Differentiating a new grid with more numerous, evenly 
distribution panels many produce a smoother data set. The control derivative discontinuities near the trailing edge of the model may be due to a greater sensitivity of the code near locations that it is enforcing the Kutta condition.

A MATLAB application was developed to systematically investigate the design and effectiveness of these mold-line changing control effectors. The application reads in the coordinates, the ADJIFORgenerated PMARC control effectiveness, and directions normal to the grid points for the ICE configuration. In this MATLAB tool, the aircraft configuration is shown with the control effectiveness derivatives interpolated over the body, similar to Fig. 6 .

The MATLAB tool allows the user to select a series of grid points and corresponding deflection heights for a proposed effector. An estimate for the effector is calculated by multiplying the ADJIFOR-generated PMARC effectiveness of each selected grid point by its specified normal displacement and then summing the products. The application also has the ability to construct an input file for PMARC with the deflected geometry. Undeflected PMARC results are subtracted from the deflected PMARC calculation to determine the proposed effectiveness of a unit displacement of the effector on forces and moments. The effectiveness of a finite displacement of these effectors is then compared to the ADJIFOR-generated PMARC data and its prediction of effectiveness.

The subsequent figures show comparisons of PMARC finite-difference calculations with the linear buildup of ADJIFOR-generated PMARC control effectiveness for the upper aircraft skin. Figure 7a shows a half span of the grid used as input to PMARC and the MATLAB tool. Some grid points are annotated to describe where on the 41-span location by 17-chord location grid the comparisons are being made. The panels affected by the deflected grid points are highlighted in gray. Figure $7 b-7 d$ shows the deflected surface finite-difference value divided by the ADJIFOR-generated PMARC estimate. Figure $7 \mathrm{~b}$ shows bump height effectiveness prediction (for location 17, 2) is not very linear with bump height, but the ratio does approach 1.0, as expected, for small displacements. The prediction of the effectiveness of spanwise extensions of deflected grid points from point 17, 2 outboard is shown in Fig. 7c. Similar chordwise extensions of deflected grid points from 17, 2 aft are shown in Fig. 7d. Both these extensions are under predicted by ADJIFOR-generated PMARC data because of the nonlinearity of the effectiveness with deflection height and the relatively large deflection used. The spanwise extensions shown in Fig. 7c were performed along row 2, very near the leading edge with a bump height of $0.2 \mathrm{ft}$. The chordwise extensions shown in Fig. 7d had a deflection of $0.2 \mathrm{ft}$ and were performed at a constant span location of column 17.
It should be noted that theses comparisons are not strictly valid because the proposed effectors represent relatively large, discrete changes to the surface, while the ADJIFOR derivatives are valid for individual, incrementally small, continuous changes in the surface shape. Nevertheless, the ADJIFOR derivatives of the three aircraft moments provide information useful to the controls designer.

Figure 8 shows the calculated control effectiveness values in terms of the change in the moment coefficients. The resulting change in moment coefficient of the aircraft $(\Delta C l, \Delta C m$, and $\Delta C n)$ due to a deflection of a single point is depicted in Fig. 8. These plots help to portray how the effectiveness of deflecting a single point to a height of $0.2 \mathrm{ft}$ varies over the upper surface of the PMARC grid. Chord location is varied while holding the span location fixed at column 17 in Fig. 8a-8c. Figure 8d-8f, conversely, varies span location while holding the chord location fixed at chord row 2 .

The finite-difference and ADJIFOR-derived effectiveness follow similar trends, with the finite-difference generally predicting greater effectiveness. Due to the similar trend information between the two estimates, the ADJIFOR-derived prediction is able to give high fidelity guidance to optimally place these effectors but not predict the effectiveness of large discrete deployments.

In a previous paper, ${ }^{7}$ the ICE configuration was shown to have satisfactory longitudinal control and damping characteristics. A preliminary stability augmentor was designed to compensate for unsatisfactory lateral-directional characteristics by using shape-morphing control effectors. In a follow-on paper, the surface description of the control effector and its maximum normal displacement may be investigated and quantified to show the feasibility of generating these moments. The original study used a shape-change device that was chosen arbitrarily with only the aid of engineering judgment. The process used in the previous study will be repeated to test the control effectors designed with information derived from an adjoint formulation of the PMARC CFD code.

\section{Conclusions}

The ADIFOR-generated CFD codes agree with the original code forces and moments. The differentiated PMARC code outputs compare well to central-finite-difference approximations with step sizes smaller than $0.5 \mathrm{deg}$. The PMARC longitudinal coefficients $C N, C A$, and $C m$, incremented with $\triangle C D_{0}$, compare quantitatively with the wind tunnel data up to 6 deg angle of attack. PMARC lateral coefficients $C S, C l$, and $C n$ compare qualitatively with wind tunnel data with a constant difference between zero and $6 \mathrm{deg}$. Wind tunnel data trends change drastically above $6 \mathrm{deg}$ angle of attack, whereas the PMARC results remain linear. 
This linear region below $6 \mathrm{deg}$ angle of attack is the primary area of interest for the initial optimization of shape morphing control effectors. CFL3D predicts a change in forces, moments, and derivatives above 6 deg angle of attack. The ADIFOR-generated CFL3D code requires lengthy execution time, but may be placed into a design loop if speed improvements can be obtained from increased use of RAM caching or an implementation of parallel code.

The ADJIFOR-generated PMARC code gave superior insight into the placement of relatively large displacement, discrete, and multi-effector shape-change control devices. ADJIFOR generally underpredicted effectiveness for these larger displacements because the code is only strictly valid over incrementally small, individual displacements, but was almost always of the correct sign.

The application of automatic differentiation to CFD codes has great potential for predicting stability and control derivatives. The calculation of these derivatives can now occur in the early design phase to influence and improve the configuration.

\section{Acknowledgements}

The authors would like to thank Thomas Zang and Jim Batterson for their support and diligent review; HPCCP for the use of their Origin 2000; Bob Weston for aid in running PMARC; Bob Biedron for his invaluable help with the CFL3D source code; Alan Carle, a developer of ADIFOR and ADJIFOR, for his guidance in implementing these tools.

Michael Park is supported by a NASA grant to George Washington University and would like to thank his advisor, Professor Robert Sandusky, for his guidance.

\section{References}

${ }^{1}$ Finley, D., "Euler Technology Assessment Program for Preliminary Aircraft Design Employing SPLITFLOW Code With Cartesian Unstructured Grid Method," NASA CR-4649, Mar. 1995.

${ }^{2}$ Charlton, E., "Numerical Stability and Control Analysis Towards Falling-Leaf Prediction Capabilities of Splitflow for two Generic High-Performance Aircraft Models," NASA CR-1998-208730, Sept. 1998.

${ }^{3}$ Bischof, C., Corliss, G., Green, L., Griewank, A., Haigler, K., and Newman, P., "Automatic Differentiation of Advanced CFD Codes for Multidisciplinary Design," Journal on Computing Systems in Engineering, Vol.3, No. 6, 1993, pp. 625-637.

${ }^{4}$ Carle, A., Green, L., Bischof, C., and Newman, P., "Applications of Automatic Differentiation in CFD," AIAA Paper 94-2197, June 1994.

${ }^{5}$ Carle, A., Fagan, M., and Green, L., "Preliminary Results From the Application of Automated Adjiont Code Generation to CFL3D” AIAA Paper 98-4807, Sept. 1998.

${ }^{6}$ Ashby, D., Dudley, M., Iguchi, S., Browne, L., and Katz J., "Potential Flow Theory and Operation Guide for the Panel Code PMARC_12," NASA Ames Research Center, Moffett Field, CA, Dec. 1992.

${ }^{7}$ Scott, M., Montgomery, R., and Weston, R., "Subsonic Maneuvering Effectiveness of High Performance Aircraft Which Employ Quasi-Static Shape Change Devices," SPIE, 1998 International Symposium on Smart Structures and Materials, paper 3326-24, pp. 223-233.

${ }^{8}$ Gainer and Hoffman, "Summary of Transformation Equations and Equations of Motion Used in Free-Flight and Wind Tunnel Data Reduction and Analysis," NASASP-3070, 1972. 
Table 1 ADIFOR and central-difference derivative comparison

\begin{tabular}{|c|c|c|c|}
\hline \multicolumn{4}{|c|}{ 1a Angle of attack derivatives } \\
\hline Delta & $C N_{\alpha}$ & $C A_{\alpha}$ & $C m_{\alpha}$ \\
\hline ADIFOR & 0.035454709062101 & -0.003267609090179 & -0.000829079154041 \\
\hline 0.00010 & 0.035454709131141 & -0.003267609093372 & -0.000829079179268 \\
\hline 0.00100 & 0.035454709061831 & -0.003267609089643 & -0.000829079157029 \\
\hline 0.01000 & 0.035454708332922 & -0.003267609023218 & -0.000829079133067 \\
\hline 0.10000 & 0.035454255577332 & -0.003267559873352 & -0.000828895343906 \\
\hline 0.50000 & 0.035452260331233 & -0.003267367165219 & -0.000828709841046 \\
\hline 1.00000 & 0.035448144567092 & -0.003266980803803 & -0.000829166439763 \\
\hline 2.00000 & 0.035426249591663 & -0.003265017618858 & -0.000828574228044 \\
\hline 3.00000 & 0.035390453908999 & -0.003261680510116 & -0.000827783833800 \\
\hline 4.00000 & 0.035340142420115 & -0.003257029240917 & -0.000826603207321 \\
\hline 5.00000 & 0.035275928055248 & -0.003251137916411 & -0.000825303188473 \\
\hline \multicolumn{4}{|c|}{ 1b Angle of sideslip derivatives } \\
\hline Delta & $C S_{\beta}$ & $C I_{\beta}$ & $C n_{\beta}$ \\
\hline ADIFOR & -0.000008538661361 & -0.000834076090398 & -0.000416633127904 \\
\hline 0.00010 & -0.000008538660777 & -0.000834076088413 & -0.000416633128362 \\
\hline 0.00100 & -0.000008538661417 & -0.000834076089846 & -0.000416633127798 \\
\hline 0.01000 & -0.000008541627900 & -0.000834068593914 & -0.000416632548211 \\
\hline 0.10000 & -0.000008540791200 & -0.000834067092253 & -0.000416631995080 \\
\hline 0.50000 & -0.000008539216973 & -0.000834039640050 & -0.000416612530736 \\
\hline 1.00000 & -0.000008539749531 & -0.000833899327802 & -0.000416547971397 \\
\hline 2.00000 & -0.000008532680933 & -0.000833404628431 & -0.000416295502544 \\
\hline 3.00000 & -0.000008525603110 & -0.000832545220898 & -0.000415871827408 \\
\hline 4.00000 & -0.000008510866398 & -0.000831363321756 & -0.000415281716613 \\
\hline 5.00000 & -0.000008493765165 & -0.000829844366410 & -0.000414523104020 \\
\hline
\end{tabular}

Table 2 Force and moment coefficients at 0 degrees of sideslip.

\begin{tabular}{|c|c|c|c|c|c|c|}
\hline Angle of Attack & $C N$ & $C A$ & $C m$ & $C S$ & $C I$ & $C n$ \\
\hline \multicolumn{7}{|c|}{ 2a Simulation database } \\
\hline 1.16 & 0.00649 & 0.01010 & 0.00454 & 0.00100 & -0.00008 & 0.00004 \\
\hline 1.62 & 0.02551 & 0.00917 & 0.00405 & 0.00096 & -0.00003 & 0.00005 \\
\hline 2.70 & 0.06726 & 0.00671 & 0.00297 & 0.00084 & 0.00010 & 0.00009 \\
\hline 3.71 & 0.10607 & 0.00385 & 0.00205 & 0.00085 & 0.00026 & 0.00008 \\
\hline 4.68 & 0.14412 & 0.00051 & 0.00126 & 0.00085 & 0.00042 & 0.00007 \\
\hline 5.66 & 0.18022 & -0.00316 & 0.00063 & 0.00074 & 0.00053 & 0.00005 \\
\hline 6.66 & 0.21742 & -0.00750 & -0.00002 & 0.00074 & 0.00064 & 0.00006 \\
\hline 7.70 & 0.25738 & -0.01238 & -0.00114 & 0.00039 & 0.00073 & 0.00007 \\
\hline 8.74 & 0.30058 & -0.01725 & -0.00216 & 0.00015 & 0.00074 & 0.00011 \\
\hline 9.67 & 0.34156 & -0.02053 & -0.00289 & -0.00062 & 0.00062 & 0.00042 \\
\hline \multicolumn{7}{|c|}{$\mathbf{2 b}$ PMARC values } \\
\hline 1.16 & 0.01178 & 0.00142 & 0.00132 & 0.00000 & 0.00000 & 0.00000 \\
\hline 1.62 & 0.02783 & 0.00054 & 0.00120 & 0.00000 & 0.00000 & 0.00000 \\
\hline 2.70 & 0.06566 & -0.00189 & 0.00077 & 0.00000 & 0.00000 & 0.00000 \\
\hline 3.71 & 0.10128 & -0.00465 & 0.00017 & 0.00000 & 0.00000 & 0.00000 \\
\hline 4.68 & 0.13564 & -0.00773 & -0.00060 & 0.00000 & 0.00000 & 0.00000 \\
\hline 5.66 & 0.17047 & -0.01128 & -0.00156 & 0.00000 & 0.00000 & 0.00000 \\
\hline 6.66 & 0.20610 & -0.01534 & -0.00273 & 0.00000 & 0.00000 & 0.00000 \\
\hline 7.70 & 0.24319 & -0.02002 & -0.00414 & 0.00000 & 0.00000 & 0.00000 \\
\hline 8.74 & 0.28026 & -0.02518 & -0.00576 & 0.00000 & 0.00000 & 0.00000 \\
\hline 9.67 & 0.31337 & -0.03019 & -0.00737 & 0.00000 & 0.00000 & 0.00000 \\
\hline
\end{tabular}

American Institute of Aeronautics and Astronautics 
Table 3 Force and moment coefficients at 2 degrees of sideslip

\begin{tabular}{|c|c|c|c|c|c|c|}
\hline Angle of Attack & $C N$ & $C A$ & $C m$ & $C S$ & $C 1$ & $C n$ \\
\hline \multicolumn{7}{|c|}{ 3a Simulation database } \\
\hline 0.00 & -0.00058 & -0.00056 & -0.00003 & -0.00190 & 0.00071 & -0.00029 \\
\hline 2.50 & -0.00047 & 0.00033 & -0.00003 & -0.00159 & -0.00050 & -0.00048 \\
\hline 5.00 & -0.00037 & 0.00121 & -0.00004 & -0.00127 & -0.00172 & -0.00067 \\
\hline 7.50 & 0.00024 & 0.00045 & -0.00011 & -0.00008 & -0.00231 & -0.00130 \\
\hline \multicolumn{7}{|c|}{ 3b PMARC Values } \\
\hline 0.00 & 0.00004 & 0.00001 & 0.00000 & -0.00087 & 0.00033 & -0.00060 \\
\hline 2.50 & -0.00006 & 0.00001 & 0.00000 & -0.00038 & -0.00081 & -0.00073 \\
\hline 5.00 & -0.00017 & 0.00002 & 0.00000 & 0.00010 & -0.00194 & -0.00086 \\
\hline 7.50 & -0.00028 & 0.00003 & 0.00000 & 0.00058 & -0.00308 & -0.00099 \\
\hline
\end{tabular}

Table 4 Force and moment coefficients -2 degrees of sideslip

\begin{tabular}{|c|c|c|c|c|c|c|}
\hline Angle of Attack & $C N$ & $C A$ & $C m$ & $C S$ & $C 1$ & $C n$ \\
\hline \multicolumn{7}{|c|}{ 4a Simulation database } \\
\hline 0.00 & -0.00010 & -0.00002 & 0.00005 & 0.00201 & -0.00062 & 0.00031 \\
\hline 2.50 & 0.00076 & -0.00019 & -0.00006 & 0.00160 & 0.00055 & 0.00049 \\
\hline 5.00 & 0.00161 & -0.00035 & -0.00017 & 0.00121 & 0.00172 & 0.00067 \\
\hline 7.50 & 0.00516 & 0.00021 & -0.00070 & -0.00059 & 0.00202 & 0.00135 \\
\hline 0.06 & 4b PMRC Values \\
\hline 2.50 & 0.00004 & 0.00001 & 0.00000 & 0.00087 & -0.00033 & 0.00060 \\
\hline 5.00 & -0.00006 & 0.00001 & 0.00000 & 0.00038 & 0.00081 & 0.00073 \\
\hline 7.50 & -0.00017 & 0.00002 & 0.00000 & -0.00010 & 0.00194 & 0.00086 \\
\hline
\end{tabular}

Table 5 PMARC force and moment coefficients with drag addition

\begin{tabular}{|c|c|c|c|c|c|c|}
\hline Angle of Attack & $C N$ & $C A$ & $\mathrm{Cm}$ & $C S$ & $C l$ & $C n$ \\
\hline \multicolumn{7}{|c|}{$5 a \quad 0$ degrees of sideslip } \\
\hline 1.16 & 0.01196 & 0.01010 & 0.00404 & 0.00000 & 0.00000 & 0.00000 \\
\hline 1.62 & 0.02807 & 0.00922 & 0.00392 & 0.00000 & 0.00000 & 0.00000 \\
\hline 2.70 & 0.06607 & 0.00678 & 0.00349 & 0.00000 & 0.00000 & 0.00000 \\
\hline 3.71 & 0.10184 & 0.00402 & 0.00288 & 0.00000 & 0.00000 & 0.00000 \\
\hline 4.68 & 0.13635 & 0.00092 & 0.00211 & 0.00000 & 0.00000 & 0.00000 \\
\hline 5.66 & 0.17133 & -0.00264 & 0.00114 & 0.00000 & 0.00000 & 0.00000 \\
\hline 6.66 & 0.20711 & -0.00671 & -0.00003 & 0.00000 & 0.00000 & 0.00000 \\
\hline 7.70 & 0.24435 & -0.01142 & -0.00145 & 0.00000 & 0.00000 & 0.00000 \\
\hline 8.74 & 0.28158 & -0.01660 & -0.00307 & 0.00000 & 0.00000 & 0.00000 \\
\hline 9.67 & 0.31483 & -0.02163 & -0.00469 & 0.00000 & 0.00000 & 0.00000 \\
\hline \multicolumn{7}{|c|}{$5 b \quad 2$ degrees of sideslip } \\
\hline 0.00 & 0.00004 & 0.00001 & 0.00000 & -0.00117 & 0.00026 & -0.00060 \\
\hline 2.50 & -0.00006 & 0.00001 & 0.00000 & -0.00069 & -0.00088 & -0.00073 \\
\hline 5.00 & -0.00017 & 0.00002 & 0.00000 & -0.00020 & -0.00202 & -0.00086 \\
\hline 7.50 & -0.00028 & 0.00003 & 0.00000 & 0.00028 & -0.00315 & -0.00099 \\
\hline \multicolumn{7}{|c|}{ 5c -2 degrees of sideslip } \\
\hline 0.00 & 0.00004 & 0.00001 & 0.00000 & 0.00117 & -0.00026 & 0.00060 \\
\hline 2.50 & -0.00006 & 0.00001 & 0.00000 & 0.00069 & 0.00088 & 0.00073 \\
\hline 5.00 & -0.00017 & 0.00002 & 0.00000 & 0.00020 & 0.00202 & 0.00086 \\
\hline 7.50 & -0.00028 & 0.00003 & 0.00000 & -0.00028 & 0.00315 & 0.00099 \\
\hline
\end{tabular}




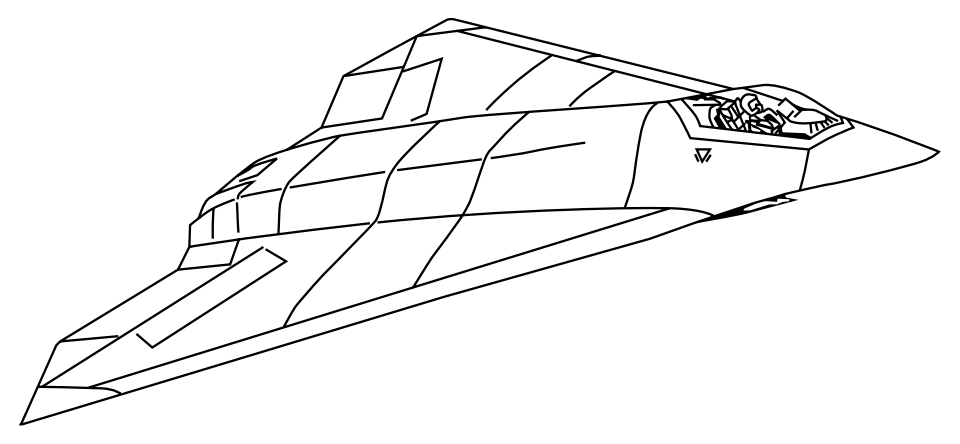

Wing Characteristics

Area ... $75.12 \mathrm{~m}^{2}\left(808.6 \mathrm{ft}^{2}\right)$

Span ... $11.43 \mathrm{~m}(37.5 \mathrm{ft})$

Aspect Ratio ... 1.74

Leading Edge Sweep ... 65 deg
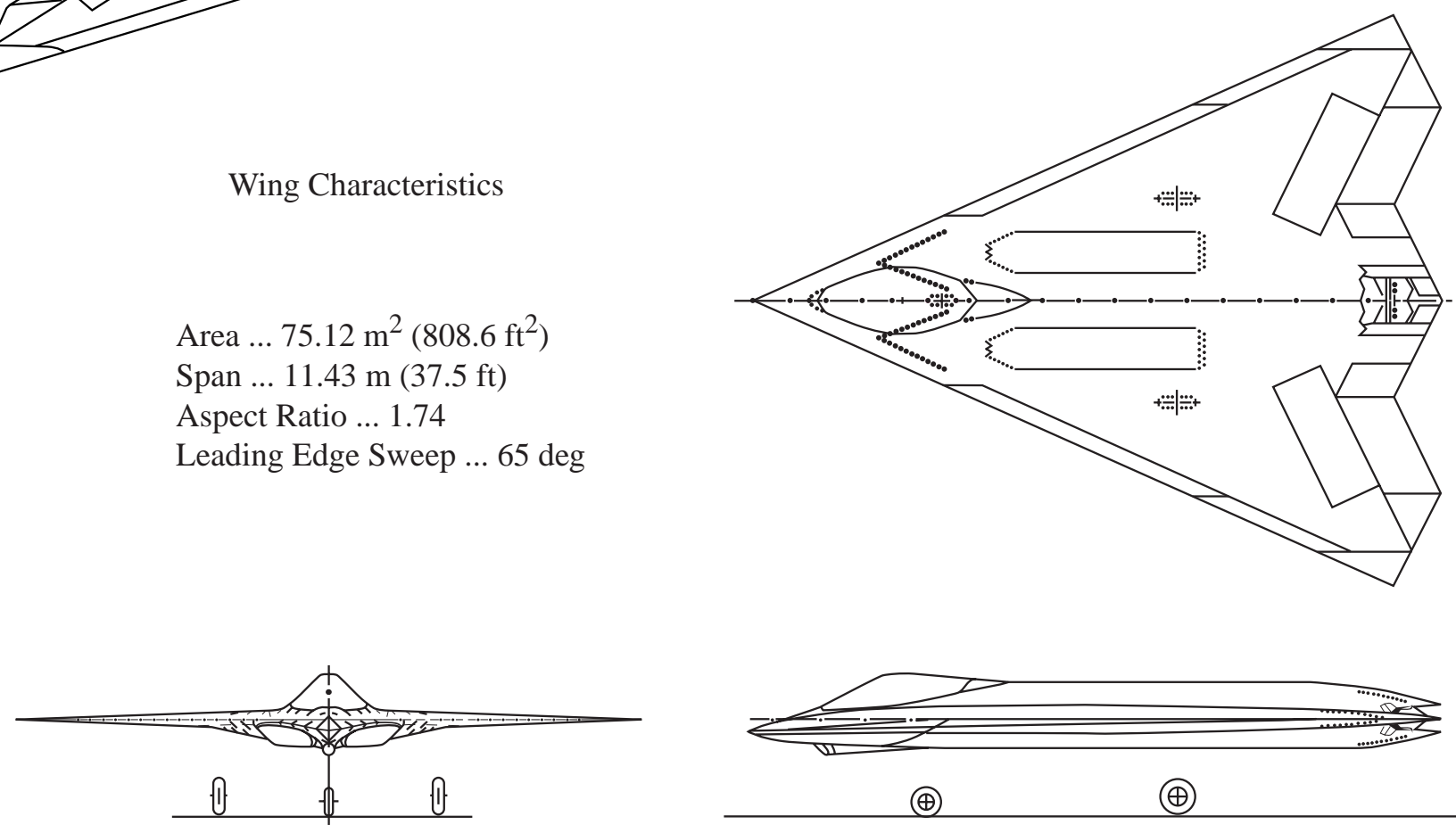

(甲)

(†)

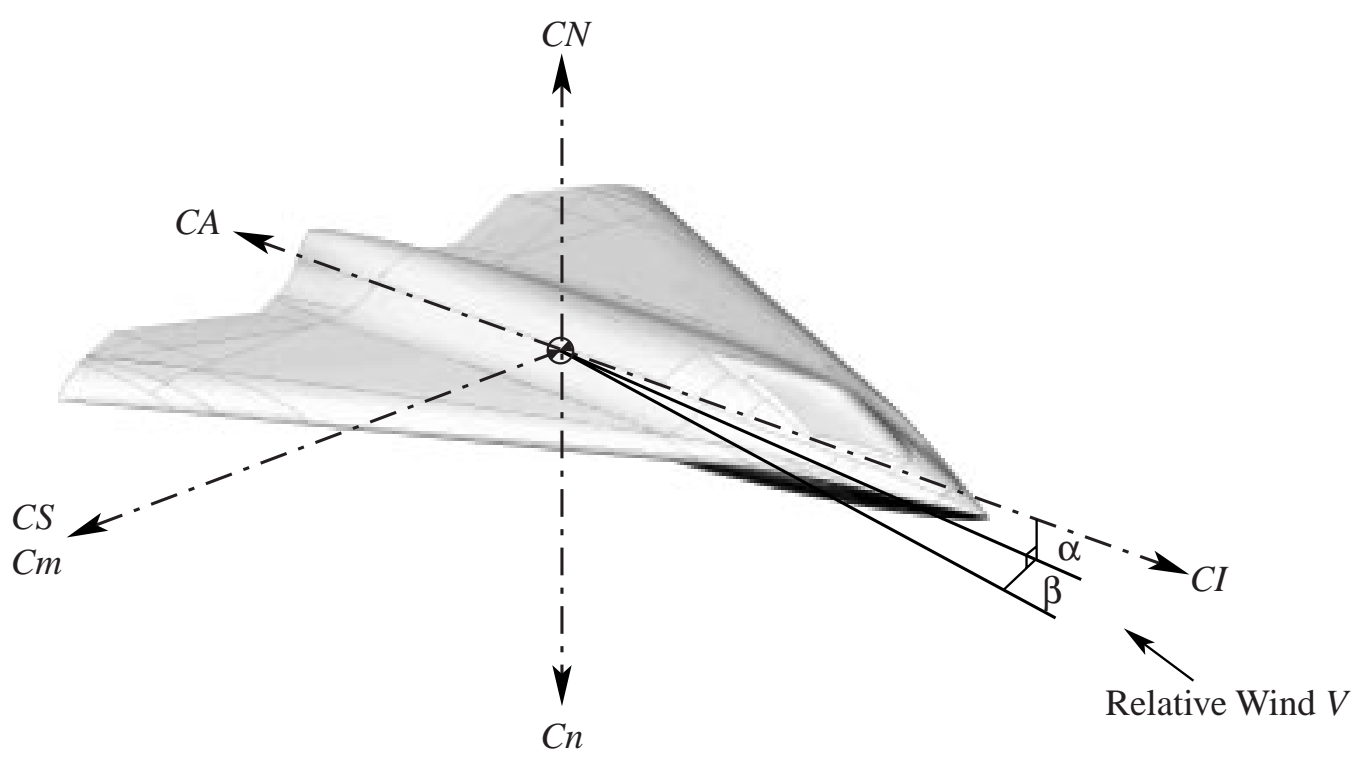

Fig. 1 LMTAS-ICE. 


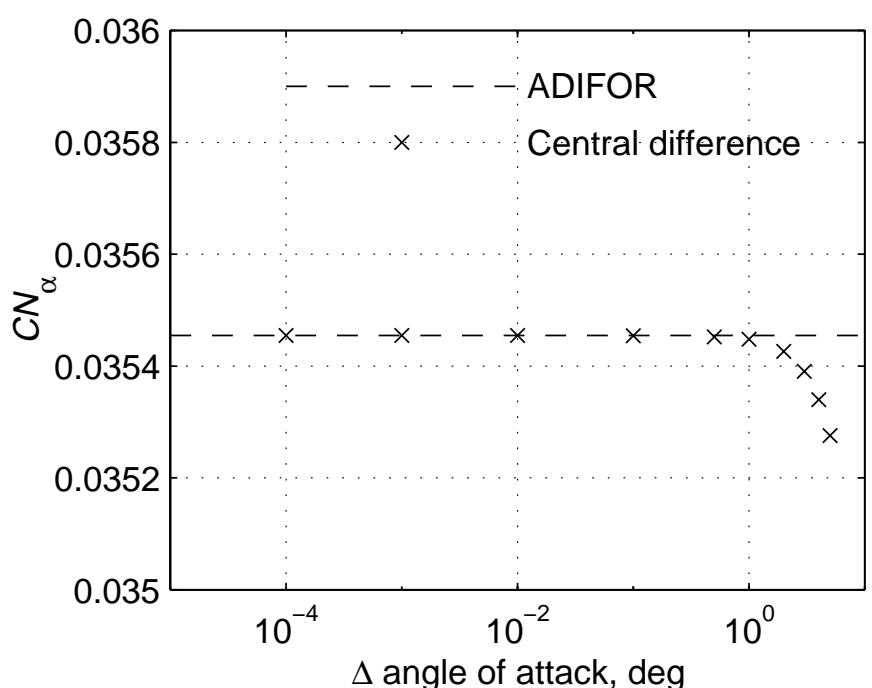

a) Normal force derivative

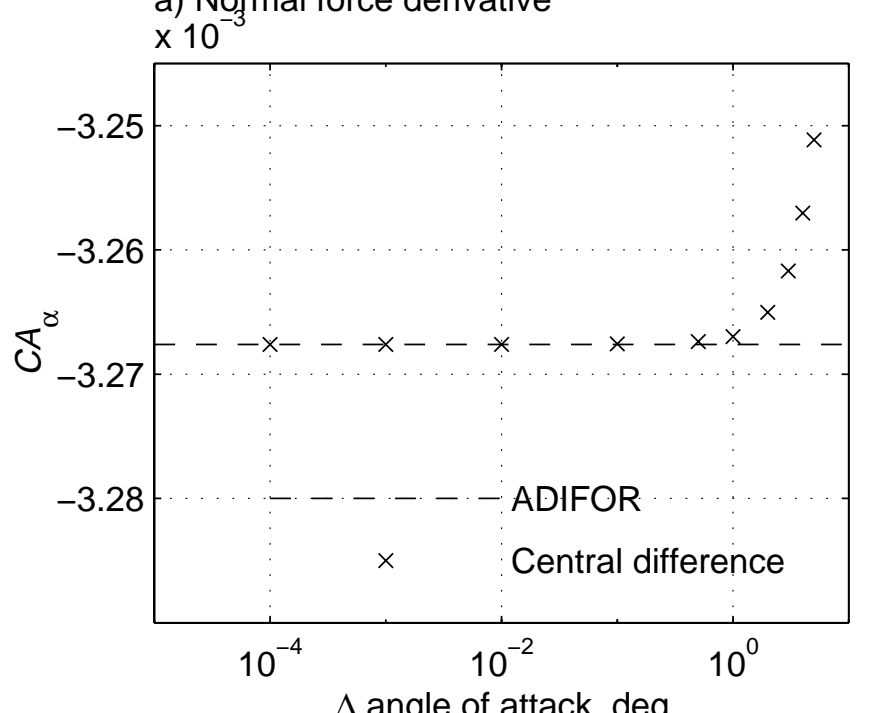

b) Axial force derivative

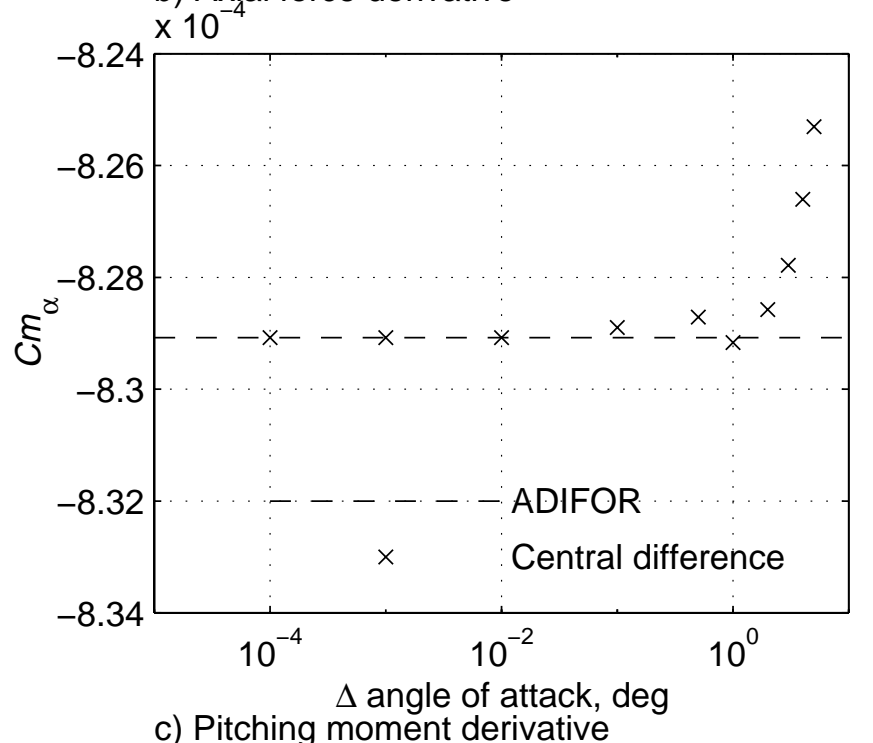

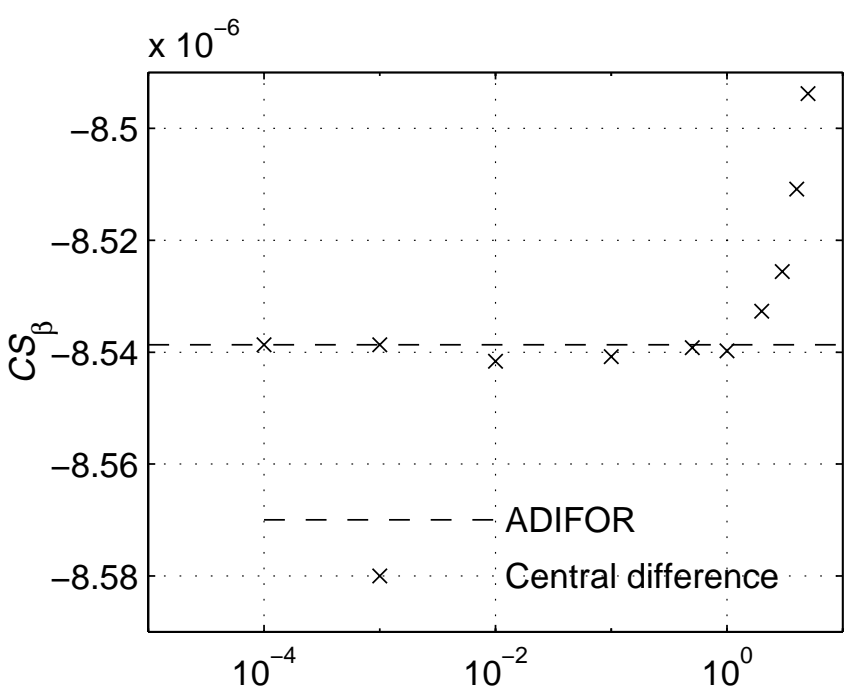

$\Delta$ angle of sideslip, deg

d) Sige force derivative

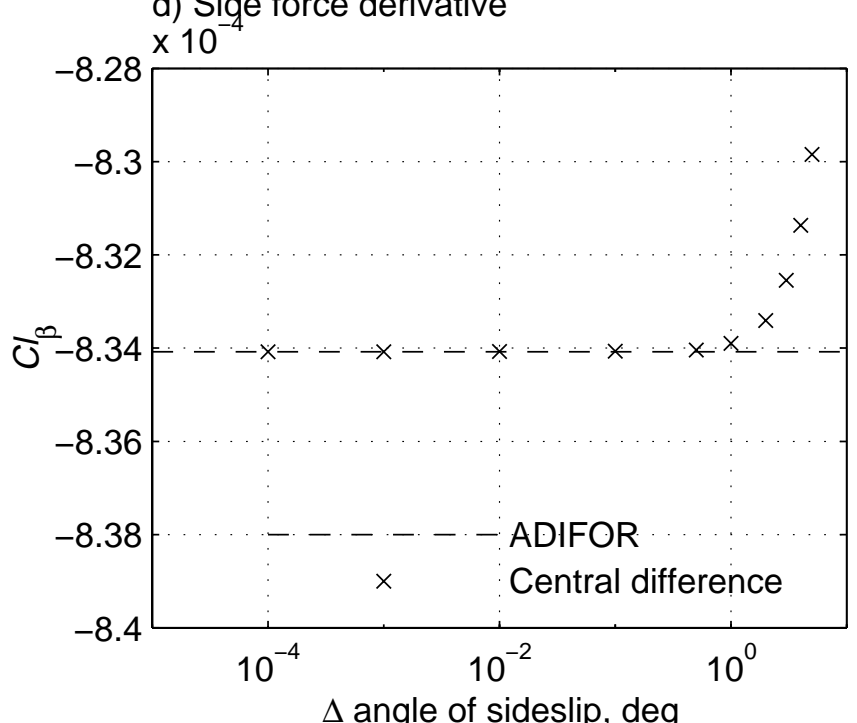

e) Rolling moment derivative

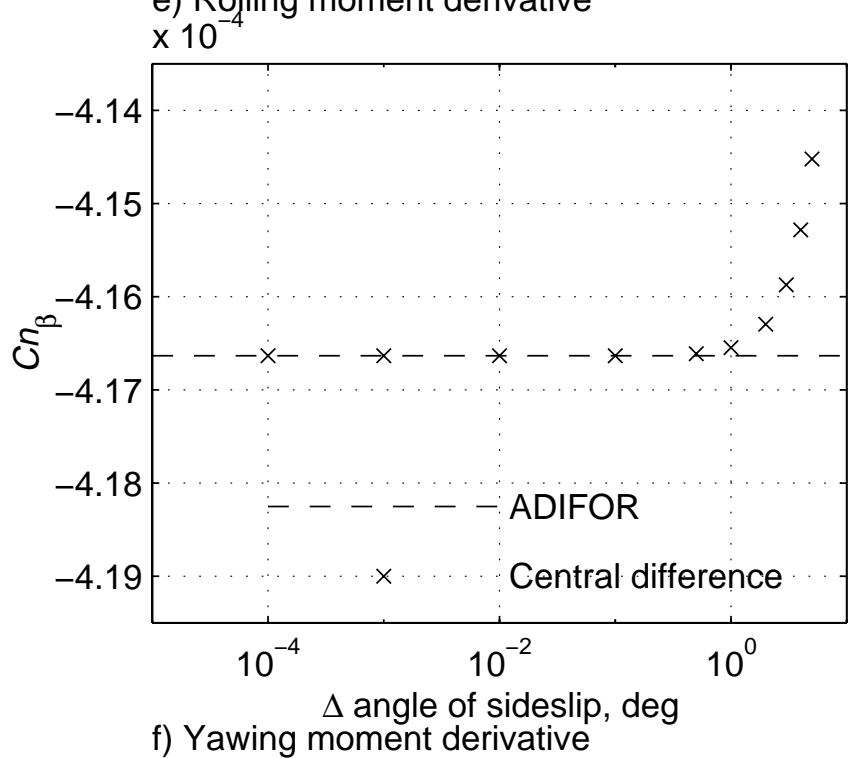

Fig. 2 Comparison of central differences to ADIFOR-generated PMARC derivatives. 


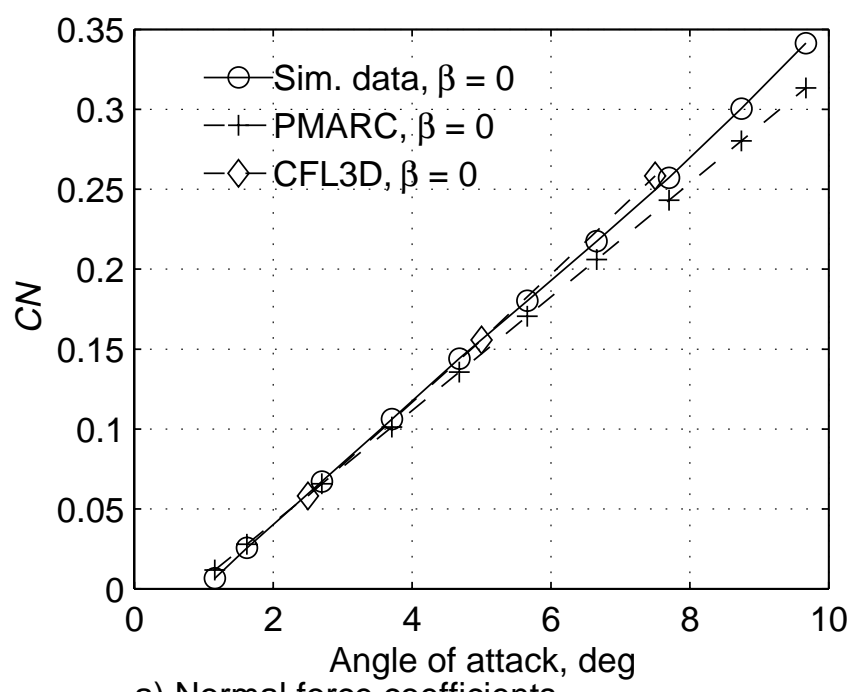

a) Normal force coefficients

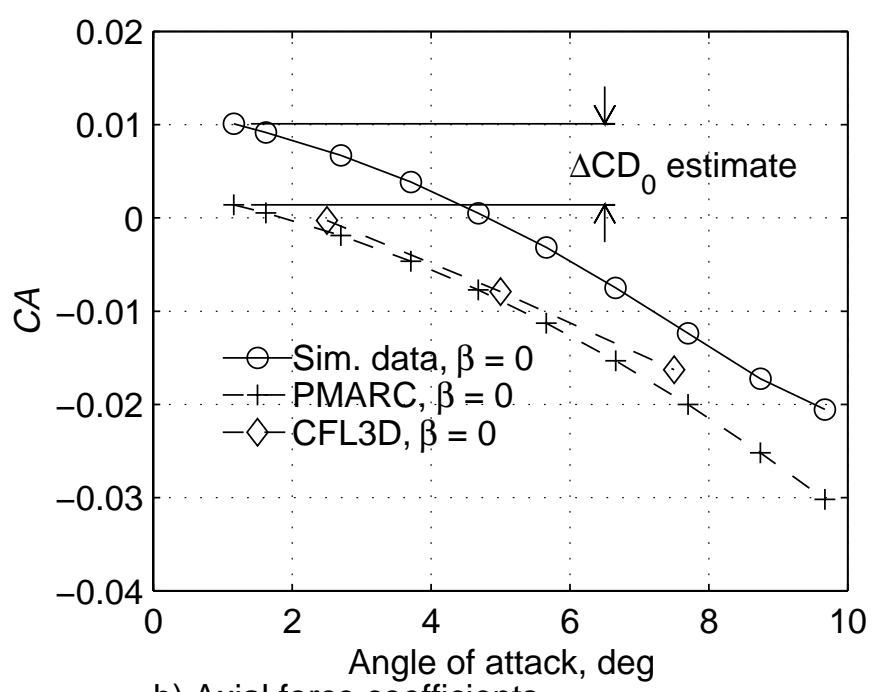

b) Axial force coefficients

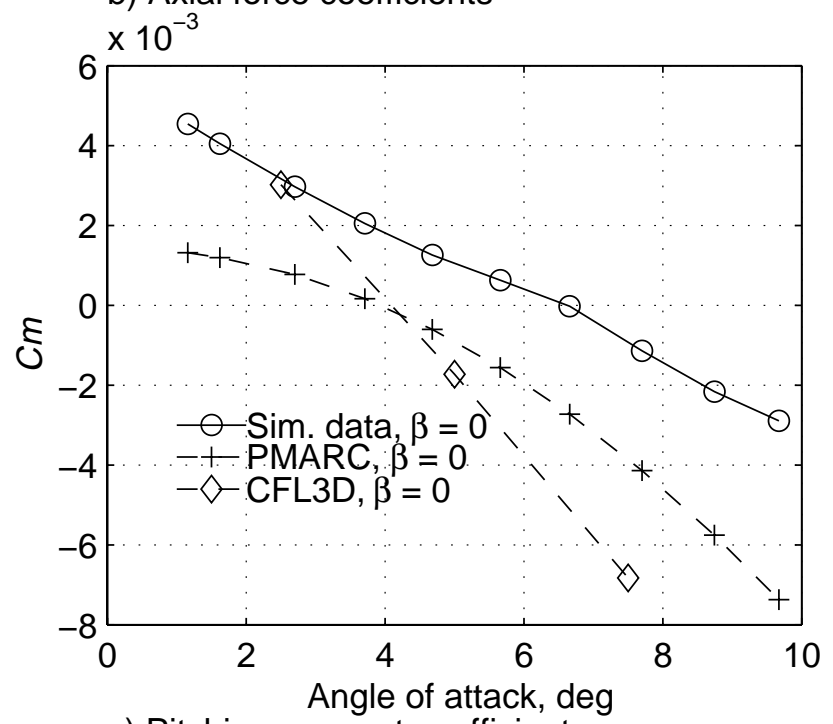

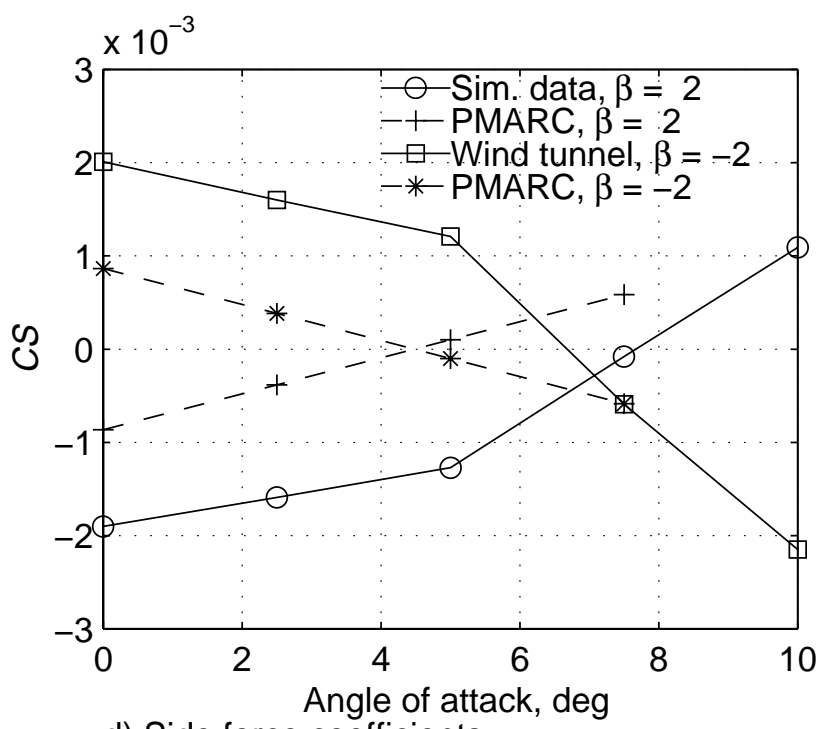

d) Side force coefficients

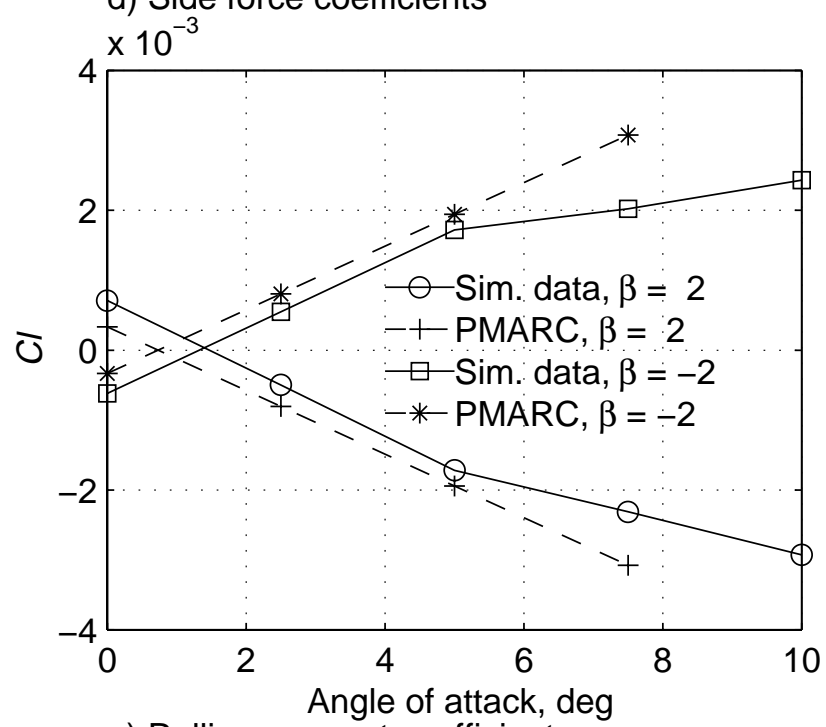

e) Rolling moment coefficients

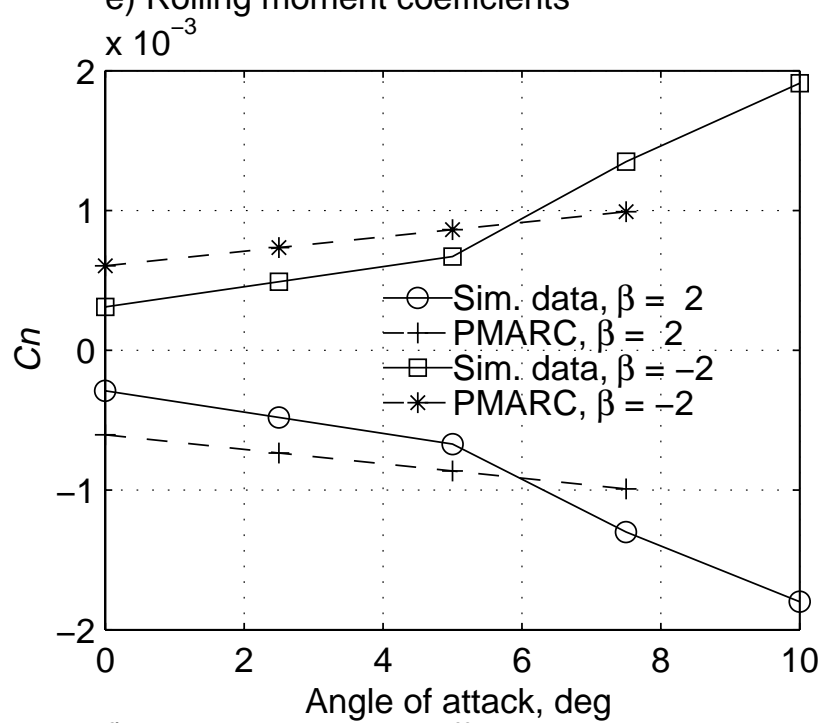

f) Yawing moment coefficients

Fig. 3 Comparison of CFD forces and moments to simulation database. 


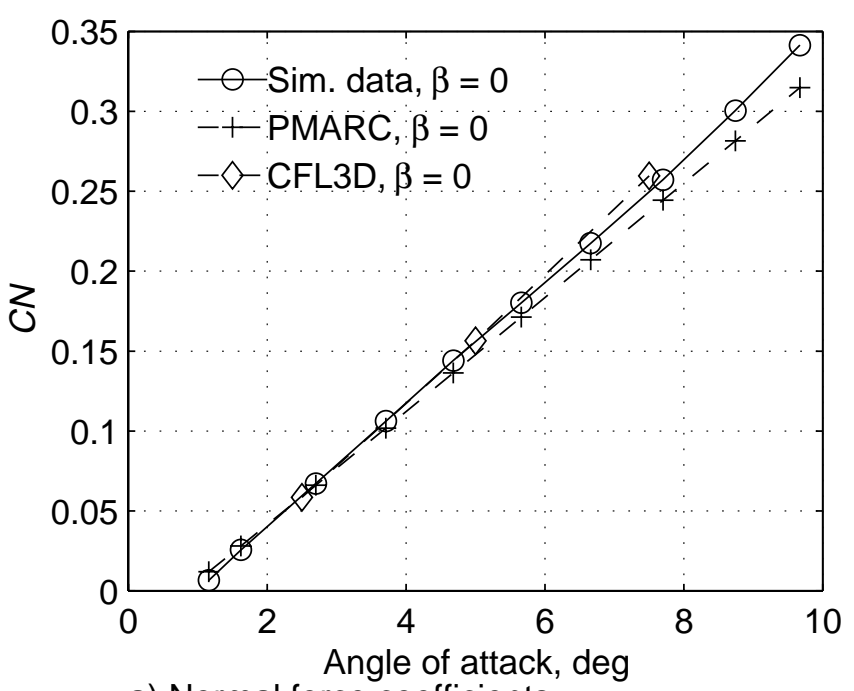

a) Normal force coefficients

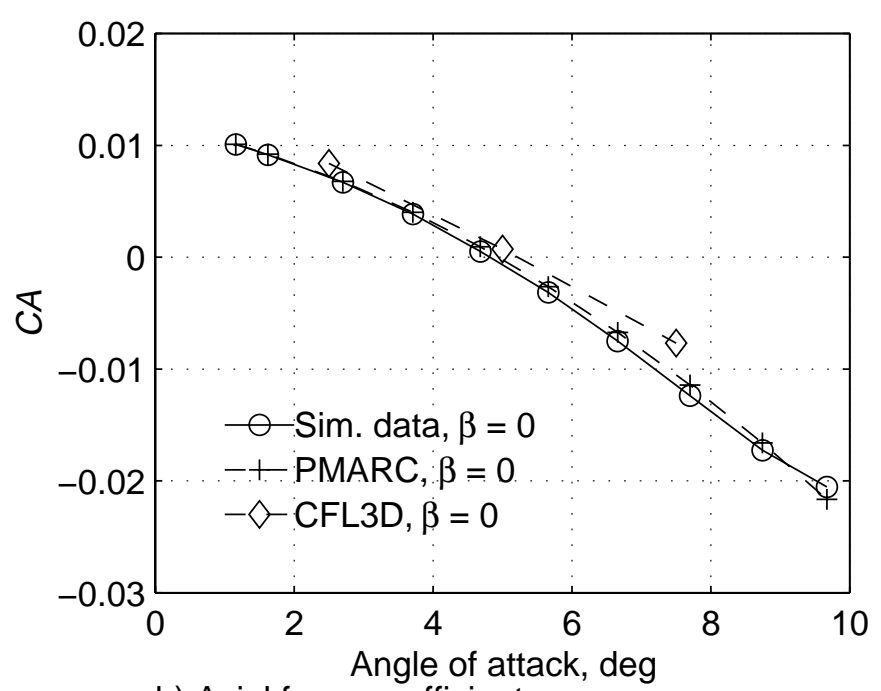

b) Axial force coefficients

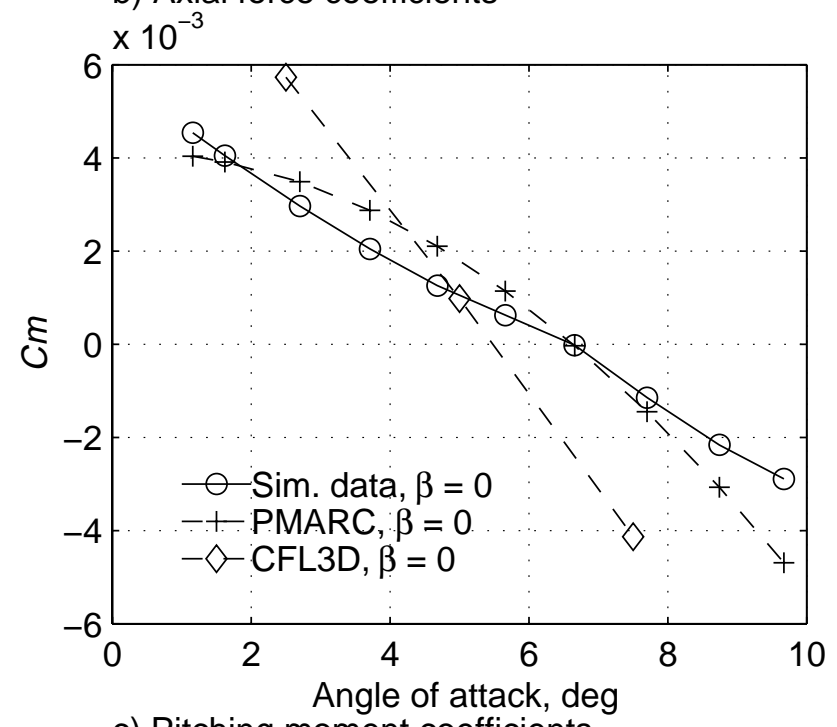

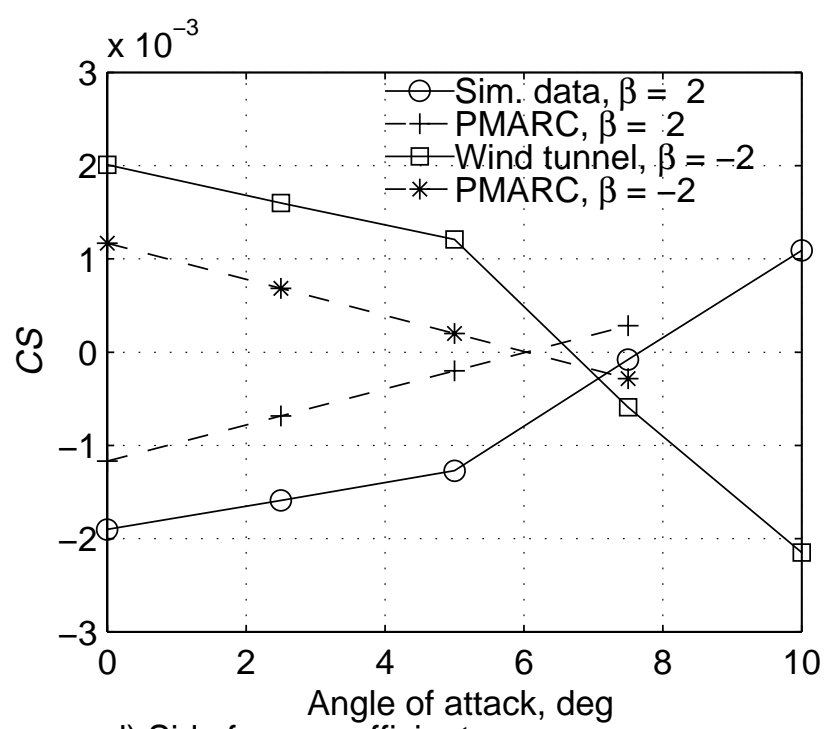

d) Side force coefficients

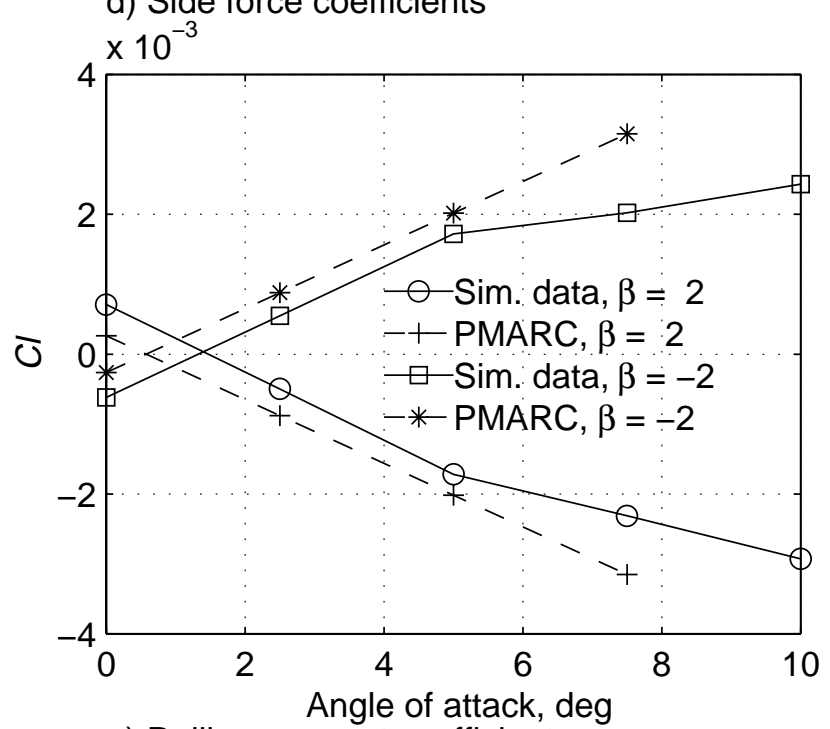

e) Rolling moment coefficients

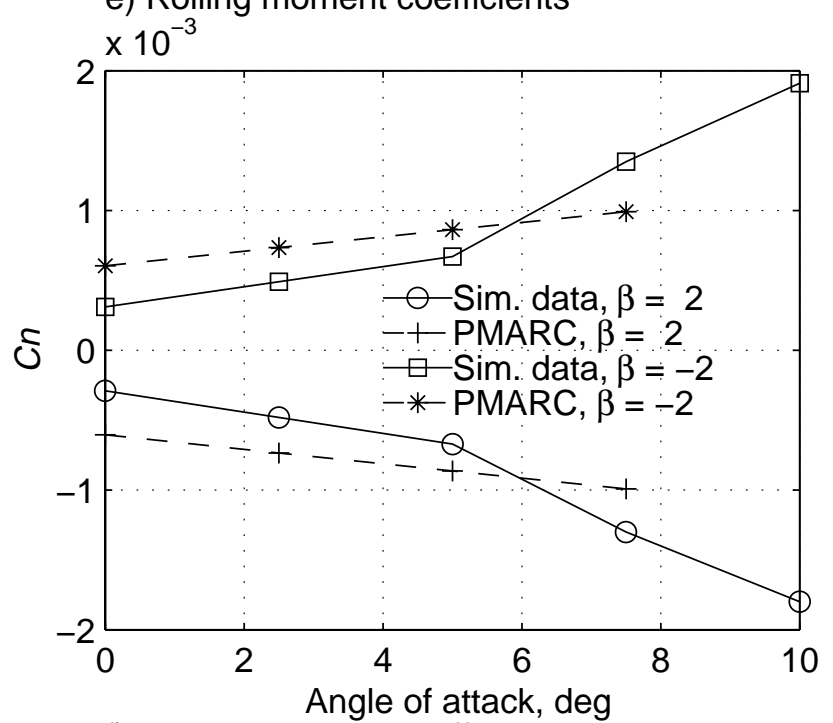

f) Yawing moment coefficients

Fig. 4 Comparison of CFD forces and moments with drag addition to simulation database. 


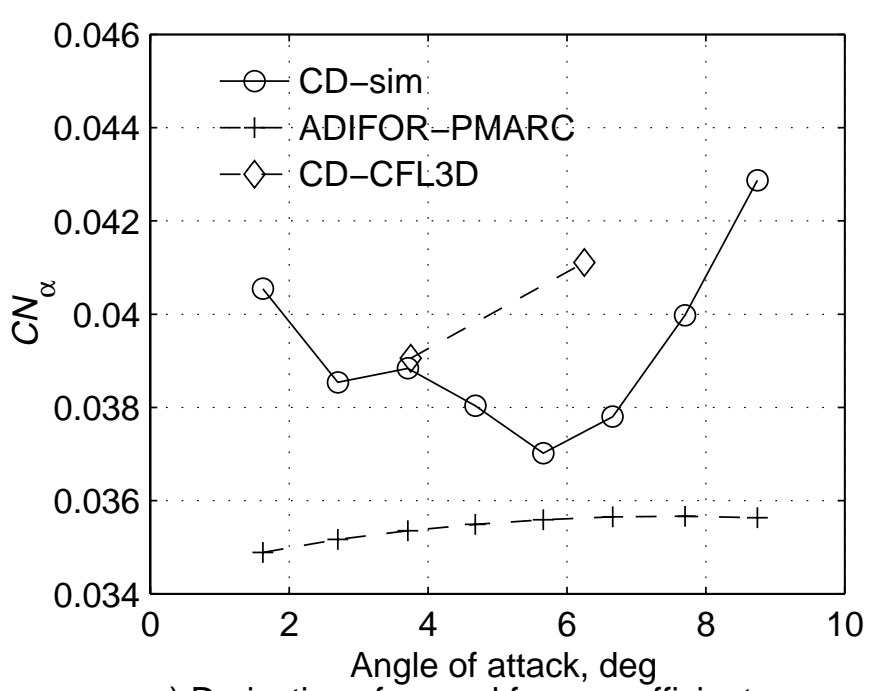

a) Derivative of normal force coefficients

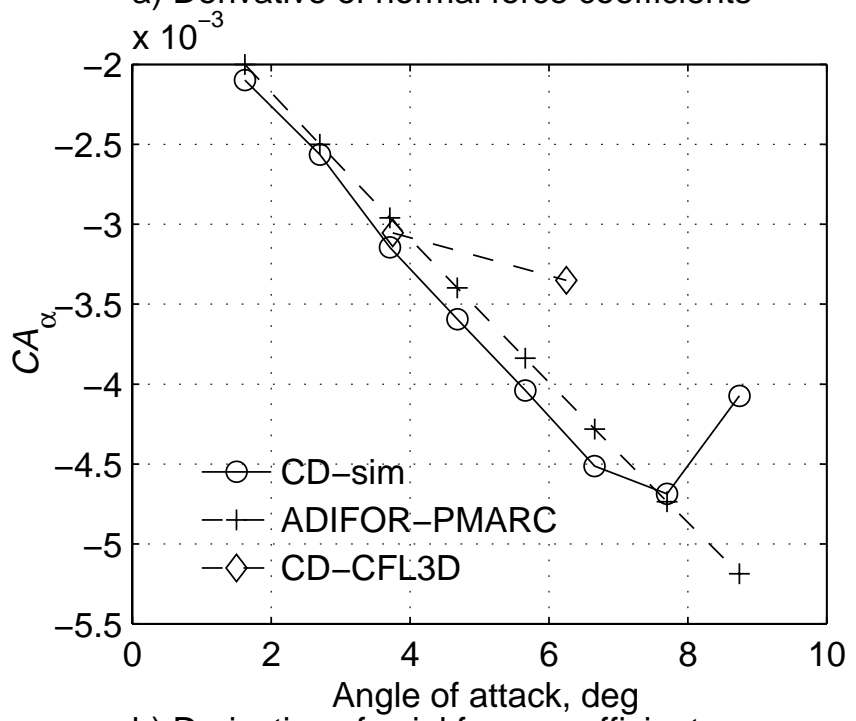

b) Derivative of axial force coefficients

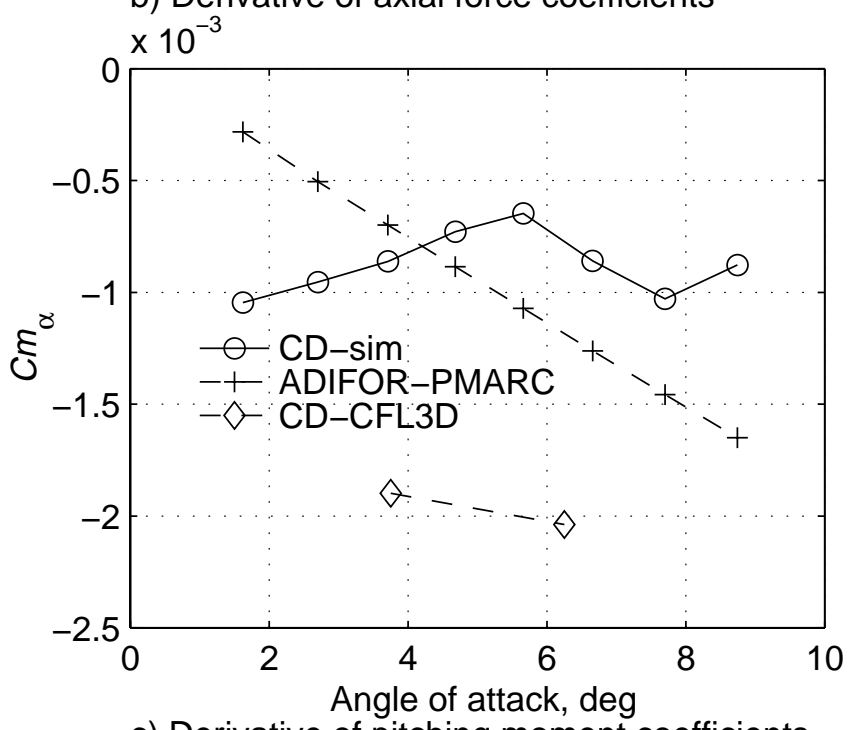

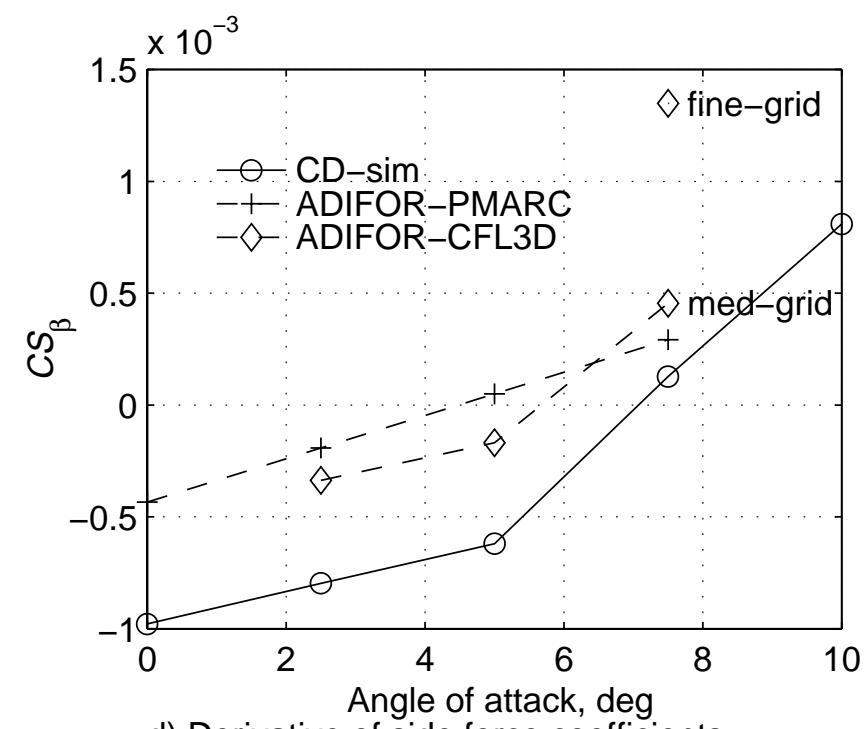

d) Derivative of side force coefficients

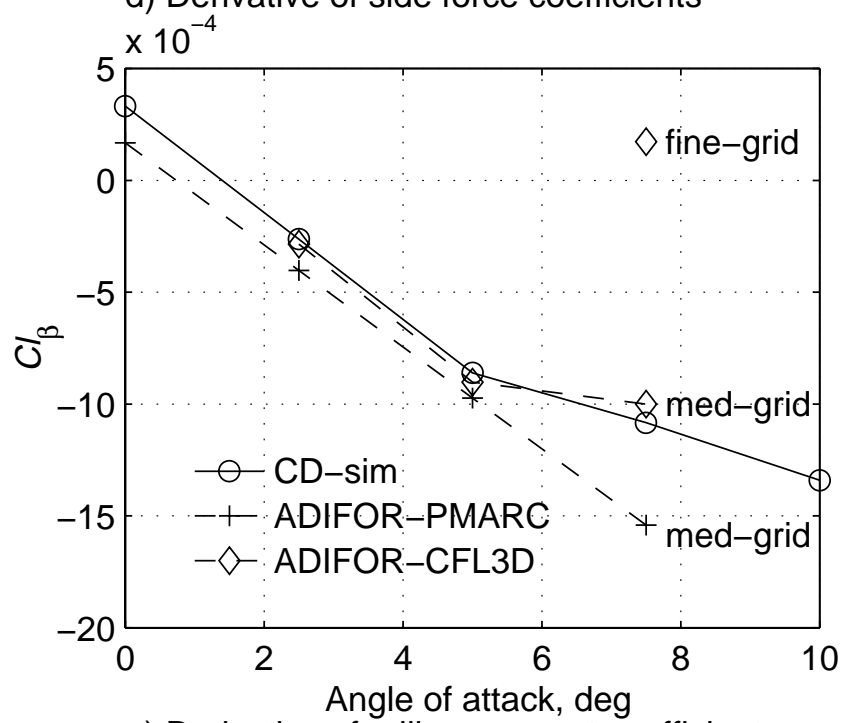

e) Derivative of rolling moment coefficients

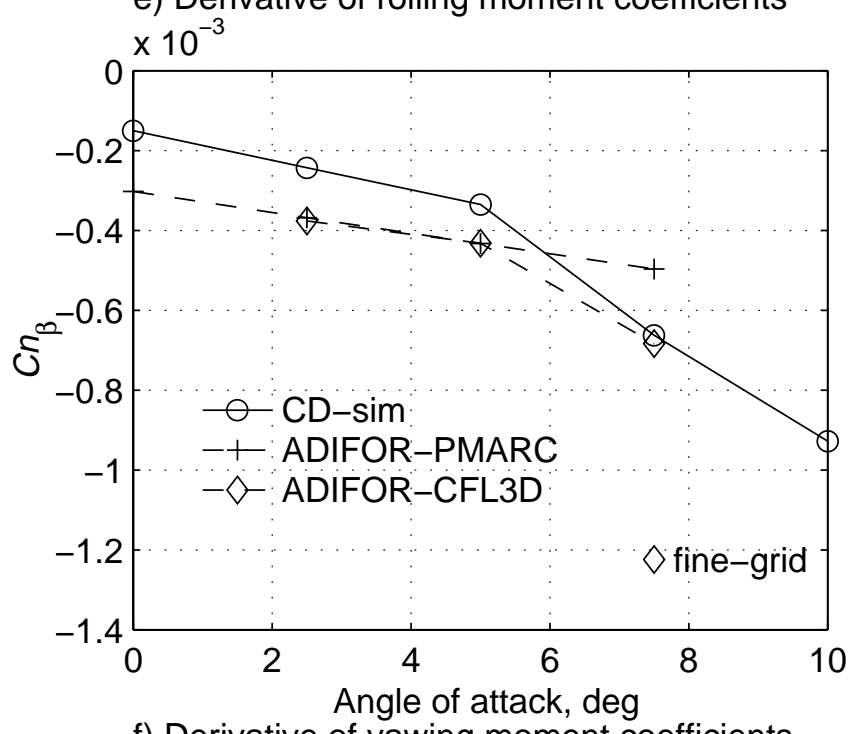

f) Derivative of yawing moment coefficients

Fig. 5 Comparison of central differences to ADIFOR-generated PMARC derivatives. 


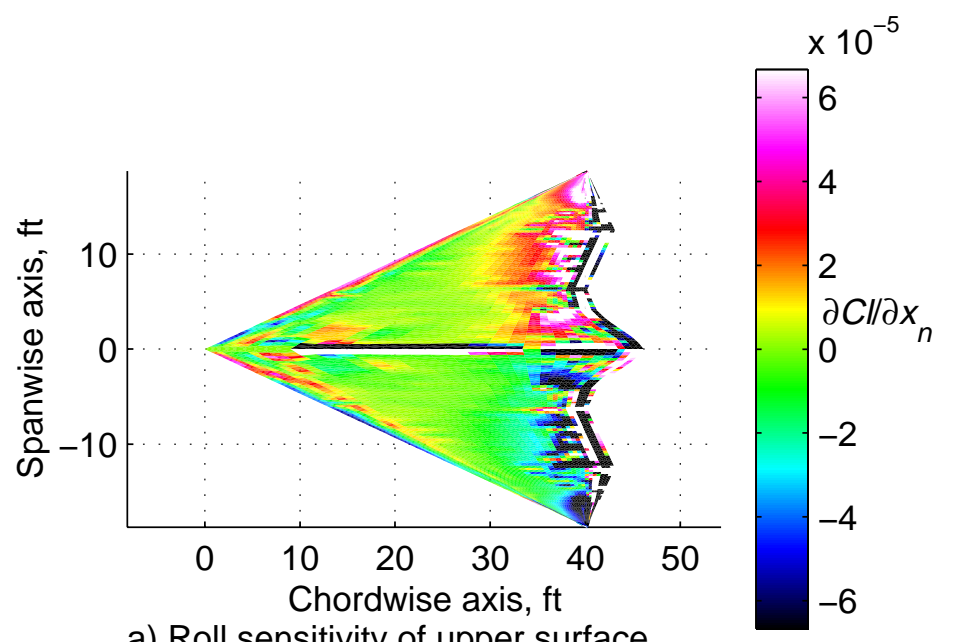

a) Roll sensitivity of upper surface

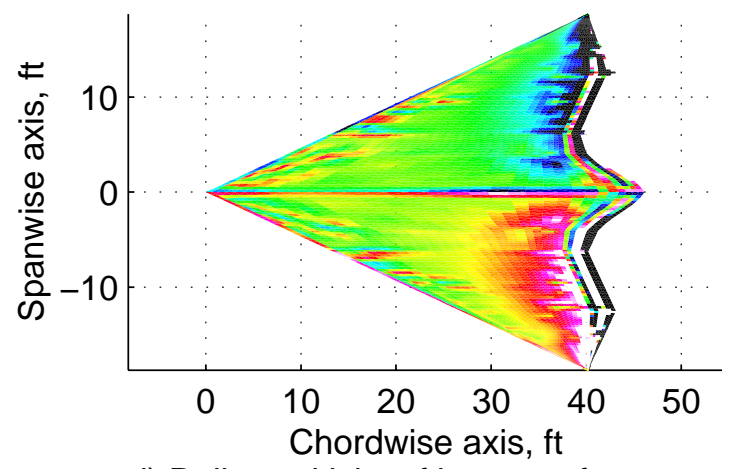

d) Roll sensitivity of lower surface
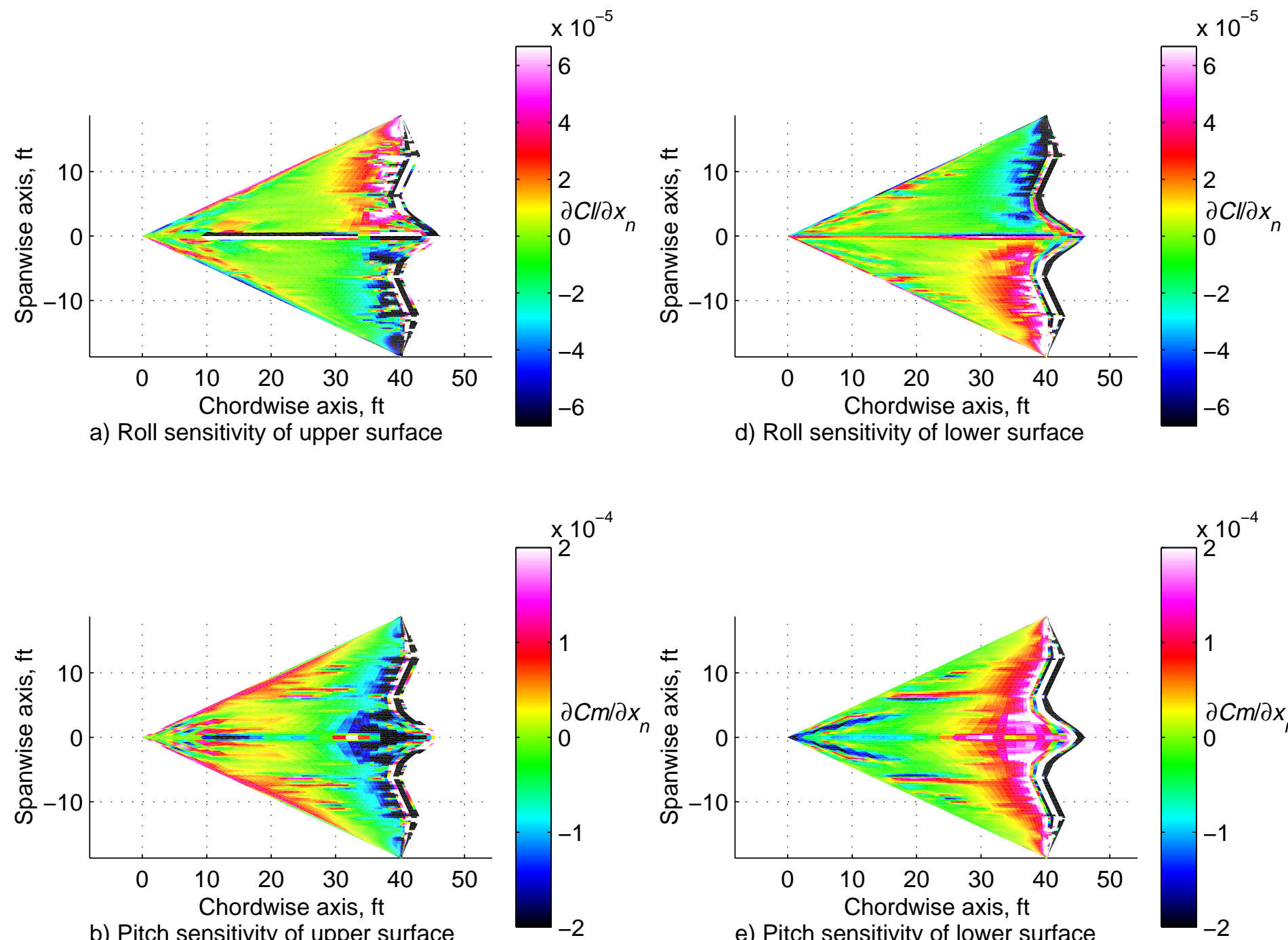

b) Pitch sensitivity of upper surface

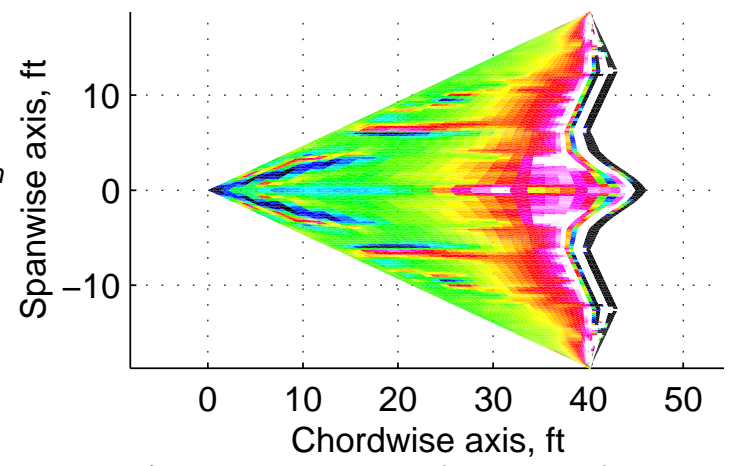

e) Pitch sensitivity of lower surface

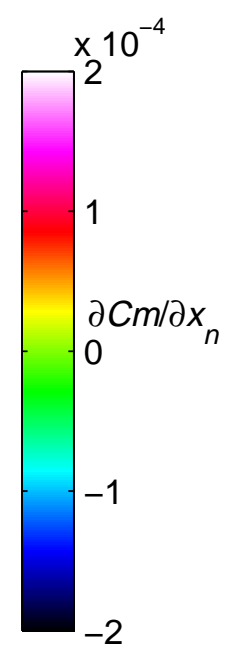

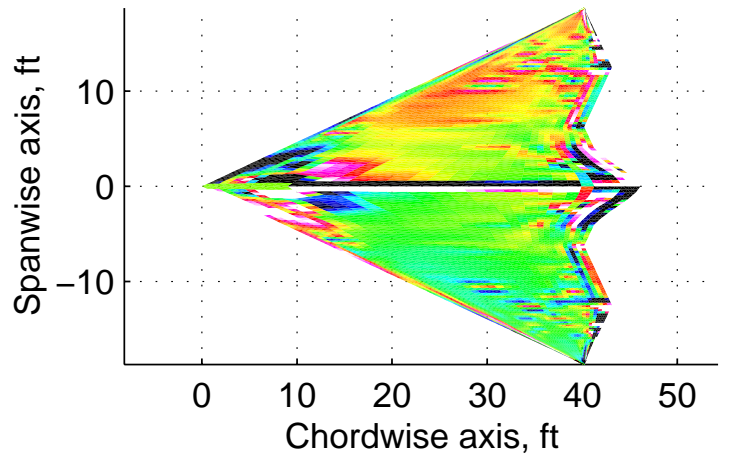

c) Yaw sensitivity of upper surface
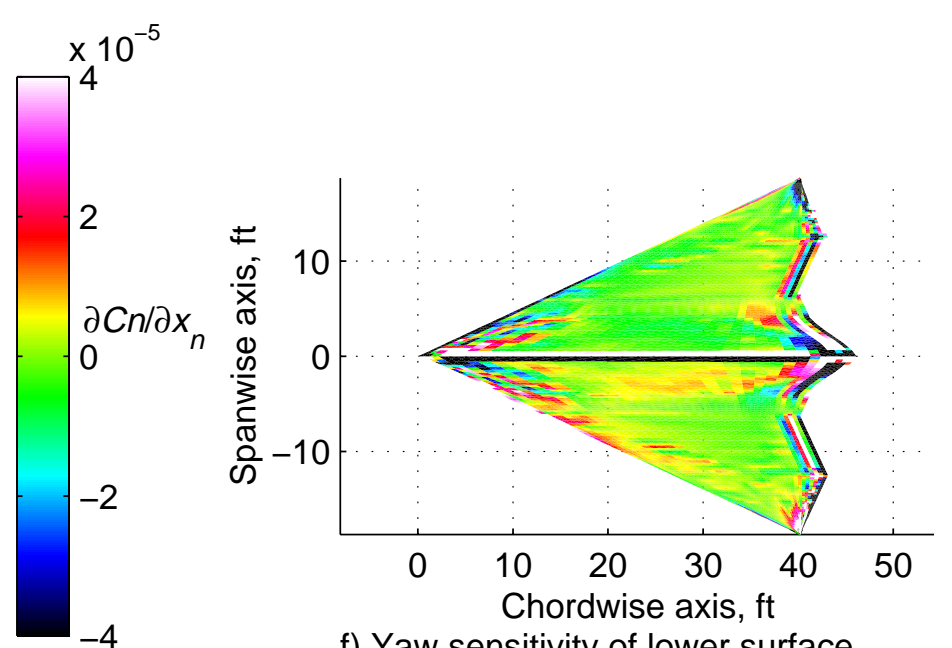

f) Yaw sensitivity of lower surface

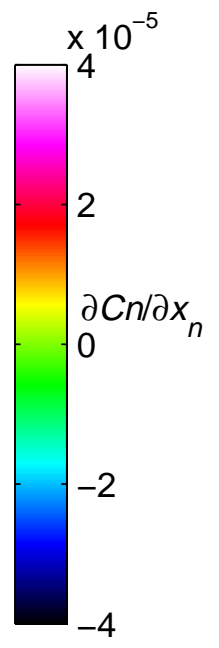

Fig. 6 ADJIFOR-generated control effectiveness contours. 


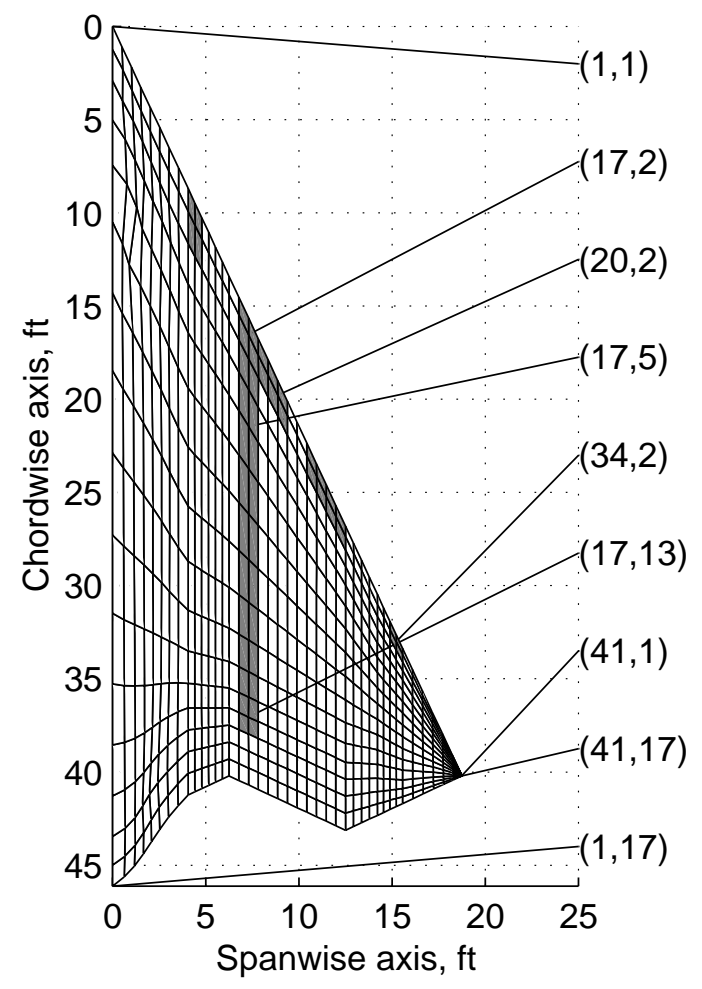

a) ICE half-span grid

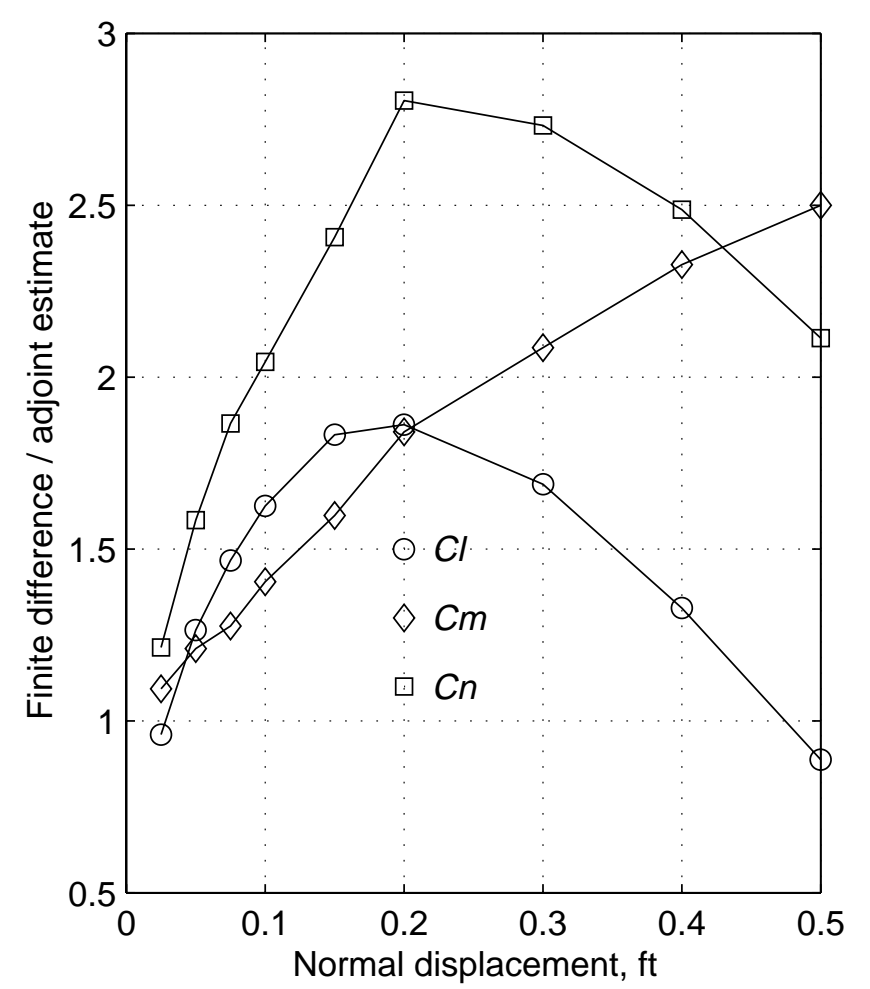

b) Variation in bump height: pt. 17,2

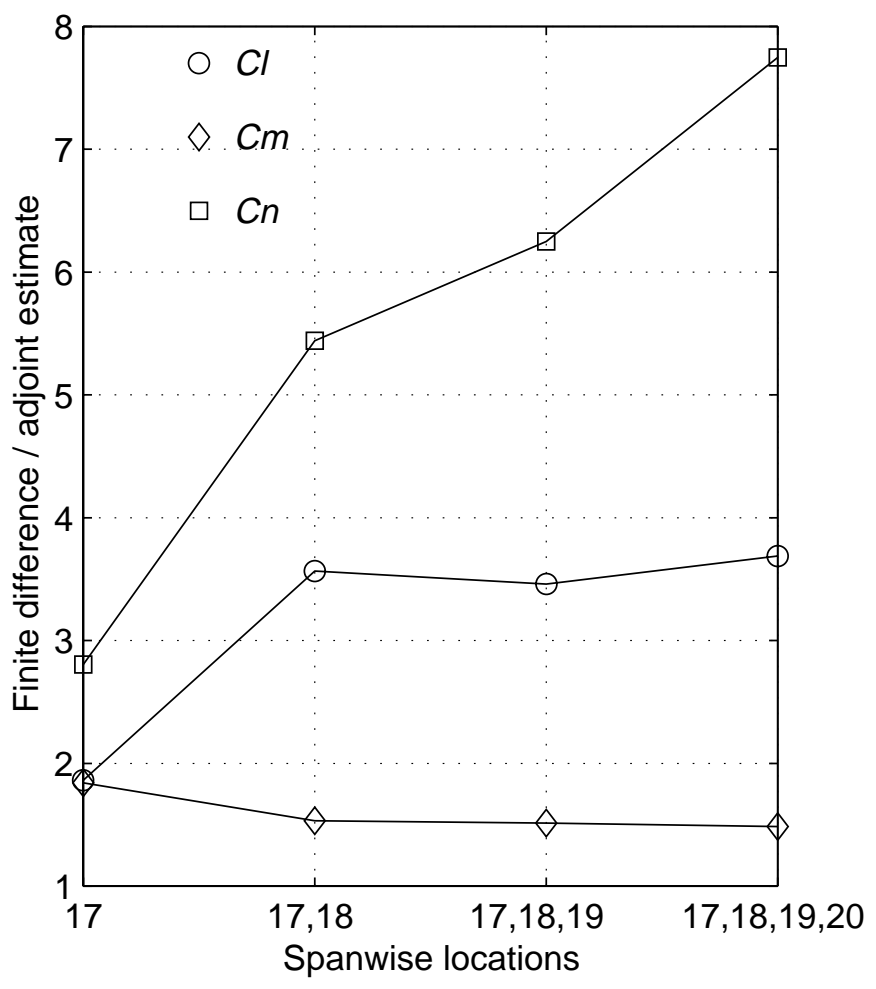

c) Deployment of spanwise adjacent bumps

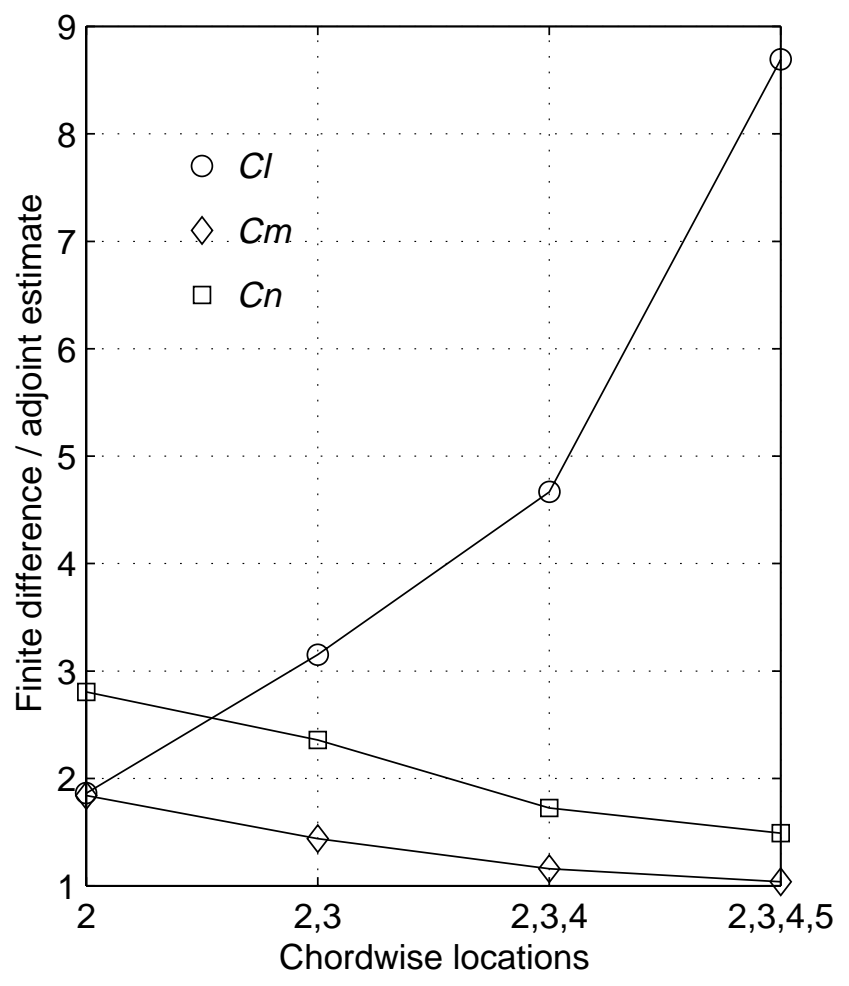

d) Deployment of chordwise adjacent bumps

Fig. 7 Comparison of ADJIFOR-generated PMARC control estimates with PMARC finite differences. 


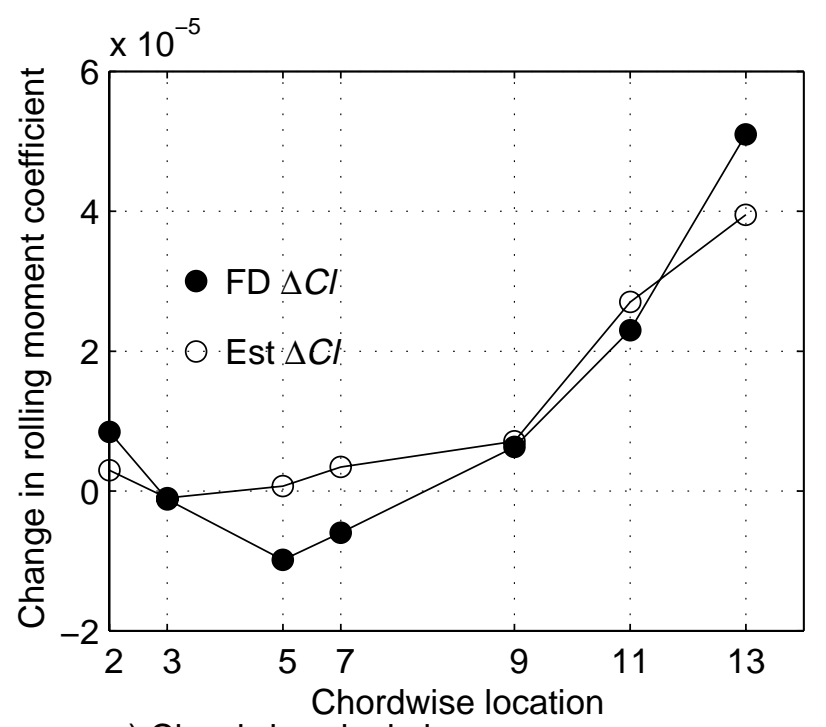

a) Chordwise single bumps

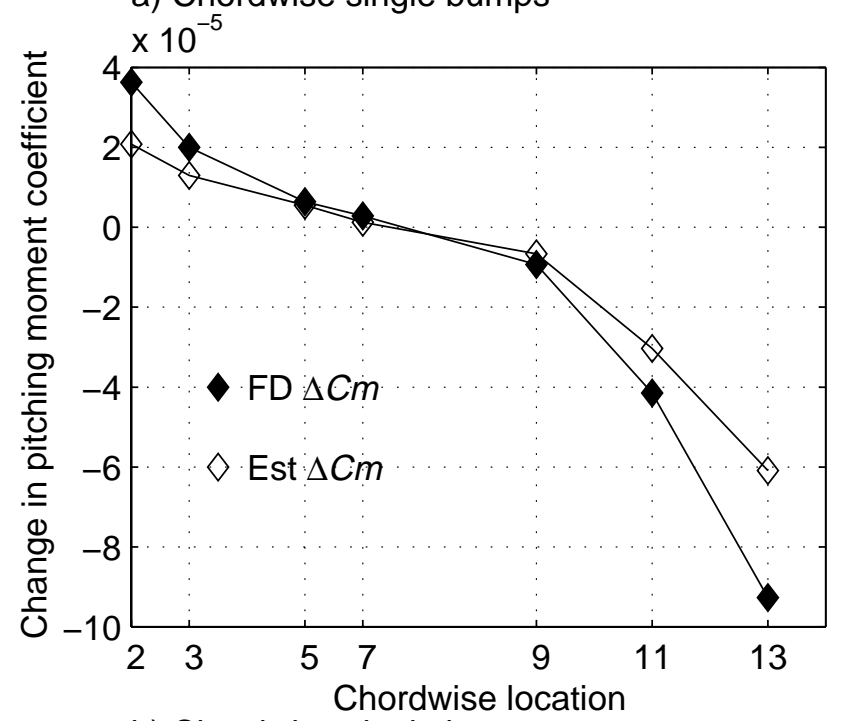

b) Chordwise single bumps

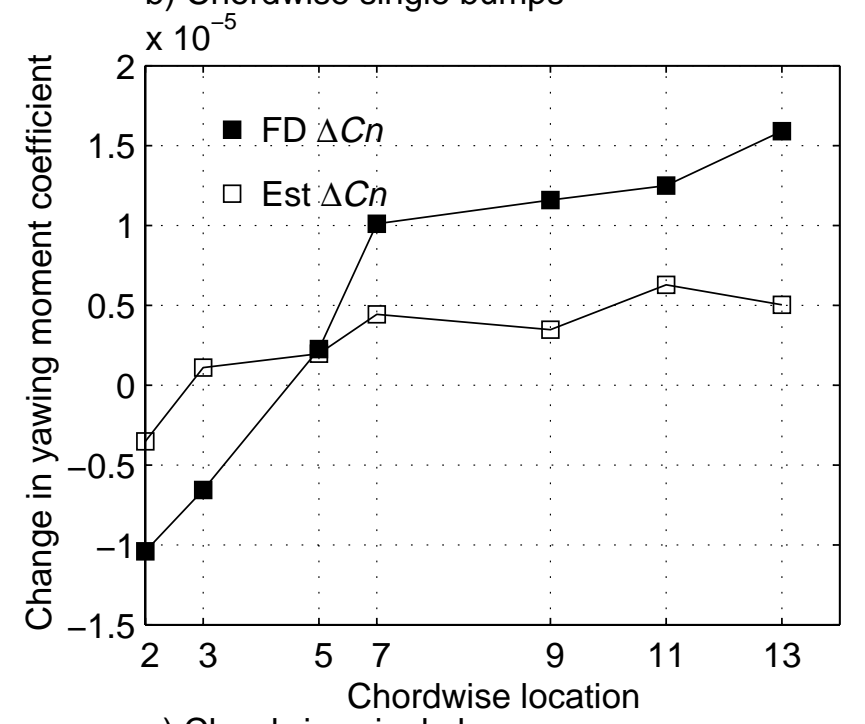

c) Chordwise single bumps

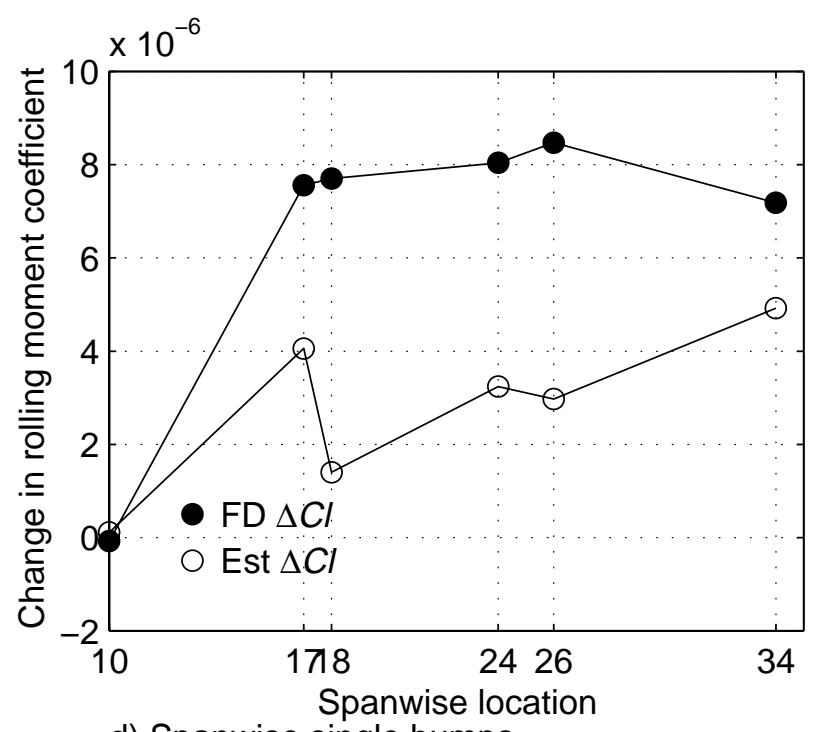

d) Spanwise single bumps

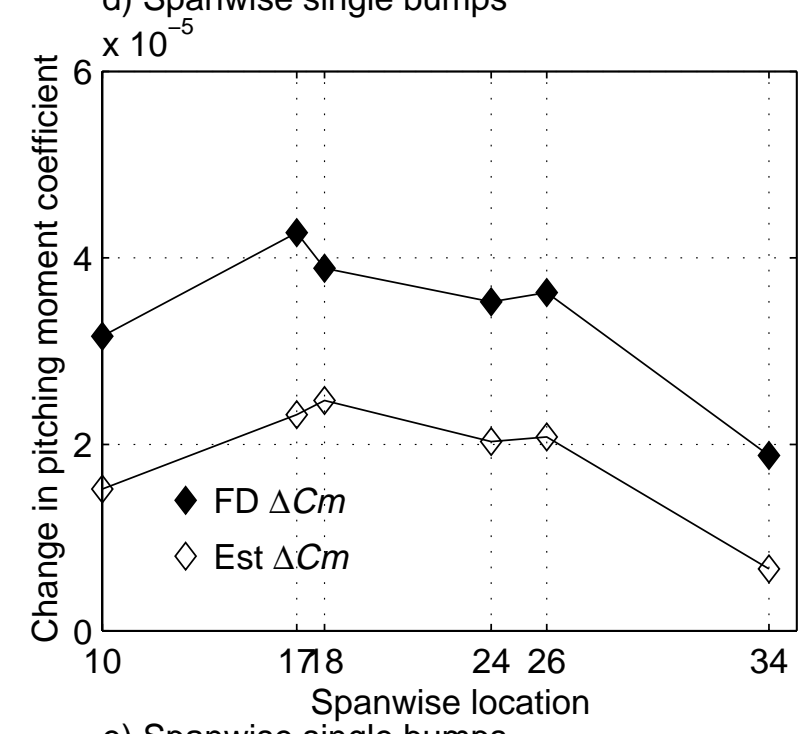

e) Spanwise single bumps

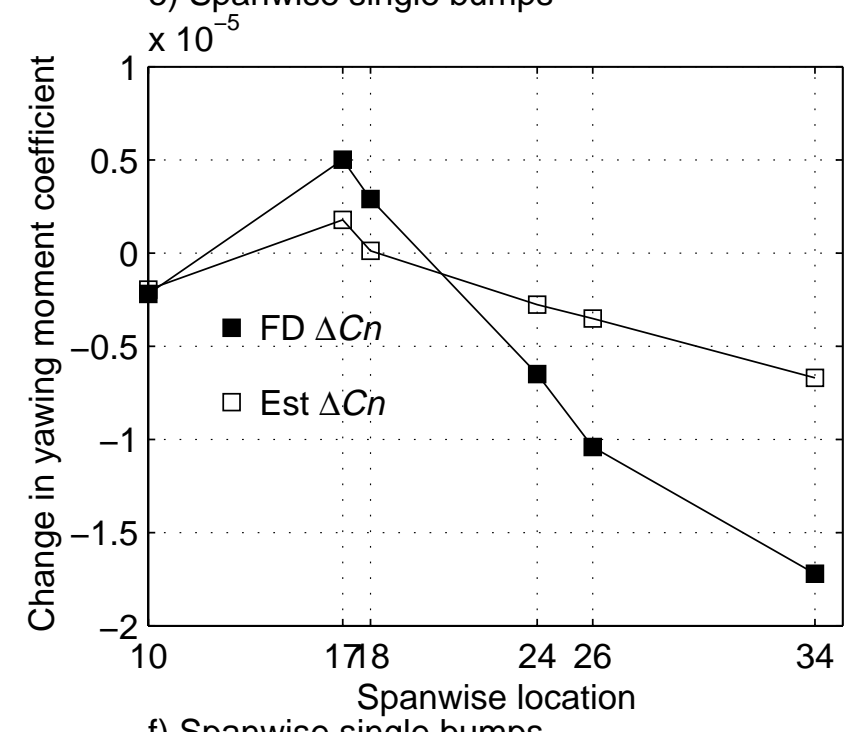

f) Spanwise single bumps

Fig. 8 Comparison of ADJIFOR-generated PMARC estimates with PMARC finite differences. 